\title{
Structural Correlations: Design Levers for Performance and Durability of Catalyst
}

\section{Layers}

Kateryna Artyushkova ${ }^{1}$, Plamen Atanassov ${ }^{1}$, Monica Dutta ${ }^{2}$, Silvia Wessel ${ }^{2}$ and Vesna

Colbow $^{2, *}$

${ }^{1}$ Chemical \& Nuclear Engineering Department, UNM Center for Emerging Energy

Technologies, University of New Mexico, Albuquerque, NM 87131

2 Ballard Power Systems, Burnaby, BC, Canada

* vesna.colbow@ballard.com

Ballard Power Systems

9000 Burnaby, BC V5J 5J8, Canada

\begin{abstract}
Durability of the catalyst layer (CL) is of vital importance in the large-scale deployment of PEMFCs. It is necessary to determine parameters that represent properties of catalysts layer and other cathode components for optimization of fuel cell performance and durability. The structure, morphology and surface chemistry of the catalyst powder affects the ionomer and catalyst interaction, ionomer dispersion in the catalyst layer and, for this reason, its morphology and chemistry. These, in turn, affect the catalyst layer effective properties such as thickness, porosity, tortuosity, diffusivity, conductivity and others, directly influencing electrode performance and durability. In this study, X-ray Photoelectron Spectroscopy and SEM are used to quantify surface species and morphology of membrane electrode assemblies (MEAs) tested under different accelerated stress test (AST) conditions. Correlations between composition, structure and morphological properties of cathode components and the catalyst layer have been developed and linked to catalyst layer performance losses. The key relationships between the catalyst layer effective properties and performance and durability provide design and optimization levers for making MEAs for different operating regimes.
\end{abstract}

\section{Keywords:}

PEMFC durability, catalyst layer chemistry, carbon corrosion, AST, structure-to-property correlations 


\section{Introduction}

Supported platinum-on-carbon catalysts remain the state of the art for fuel cells today, but more information is needed about the factors of activity and durability. Durability is the determining factor in the useful lifetime of polymer electrolyte membrane fuel cell (PEMFC) systems. Fuel cells must be resistant to changes in morphology and surface properties. There are, however, significant changes in catalyst structure and properties during operation.

The U.S. DOE have established durability-test protocols, which includes tests for electrocatalysts, electrocatalyst supports, chemical and mechanical tests of membranes. [1] Ideally, a component designer would like to evaluate new materials and cell performance with a minimum of long-term PEMFC testing. Accelerated stress tests (ASTs) have become the standard for PEMFC aging protocols.[1, 2]The use of ASTs has been shown to be very useful for assessing the durability of the PEMFC components. Performance losses and component damages can be more efficiently analyzed under specific working conditions than applying costly and time-consuming steady-state lifetime tests.[2] By analyzing the degradation data of individual cells, Bae et.al illustrated a methodology for estimating the lifetime of the PEMFC stack using the statistical theory of the smallest order. [3] The use of segmented cells enabled to understand the origin of local degradations in PEMFC. [4]

There is a critical need for creating a set of structural metrics reflecting the properties of catalyst layer components, such as support, catalyst, ionomer, etc., that can be used for sound prognoses of activity and durability. The use of ASTs and the metrics reflecting the performance losses and degradation of components has been widely utilized, but there is a missing link between AST parameters and chemical and morphological changes that occur during ASTs and, what's even more important, there is a lack of a direct relationship between the initial structure of the catalyst layer components and durability metrics used. [5]

Surface chemistry and structural morphology of individual components are critical for mass transport properties, water and heat management and subsequently electrocatalytic activity and durability. Carbon is used in fuel cells as an electrocatalyst support to ensure electronic conductivity between the electrocatalyst and the current collector. The carbon support has defects, dislocations, and discontinuities at the edges of layer planes. These 
defects tend to chemisorb oxygen, giving rise to surface functional groups, which in turn determine the surface chemistry of the carbon blacks. The surface chemistry, in turn, affects the hydrophobicity and thus wetting of products and reactants as well as dispersion in aqueous inks. The topography of real solid surfaces plays an important role in defining the electronic energy distribution at surface sites, particularly when irregularities at the atomic level are taken into account. Likewise, surface irregularities at the nanometric level determine the electrocatalytic properties. [6]

The importance, of deriving a set of design criteria for benchmarking potential fuel cell electrocatalysts through ex-situ durability tests assessing stability and degradation processes, cannot be overlooked. [7] The loss of electrochemically active surface area, degree of Pt dissolution, Pt particle growth and carbon oxidation are among most often evaluated parameters. [8] We will discuss the existing understanding related to chemical and morphological properties derived from studies of (1) Pt electrocatalysts; (2) carbon supports and (3) Nafion-based ionomer, as these three represent key components of the catalyst layer in catalysts coated membranes (CCM). [4, 5, 9, 10]

I. Pt electrocatalyst. In many studies of PEMFC, it was shown that catalyst degradation is one of the major reasons for gradual performance losses directly related to a loss of accessible surface areas of the active catalyst components. The three fundamentally different mechanisms of loss of electrochemically accessible surface area (ECSA) of Pt are Pt dissolution, the migration and coalescence of Pt nanoparticles on the support, and detachment of nanoparticles from the support. [10] There is a growing consensus that the platinum dissolution is a major factor limiting the lifetime of polymer electrolyte fuel cells, especially under varying load conditions and at the high potentials of the cathode. The analysis of the catalyst after the AST revealed that the well dispersed Pt particles of the original electrode form irregular and branched agglomerates in the catalytic layer. [2] Ex situ transmission electron microscopy (TEM) of catalyst layers (CLs) after long-term steady-state and potential cycling operation has shown dramatic changes in platinum particle size and distribution. Two groups of particles were observed in TEM: spherical particles still in contact with the carbon support and nonspherical particles removed from the carbon support. There are also reports on the formation of PtO. X-ray photoelectron 
spectroscopy (XPS) has provided evidence that Pt(IV) can be formed via oxidation of Pt(II) by hydrogen peroxide.

II. Carbon support. Carbon black is widely used as an electrocatalyst support but still suffers from issues that decrease the catalytic activity of the material. [9] In addition to the loss of the platinum, the carbon support that anchors the platinum crystallites and provides electrical connectivity to the gas-diffusion media and bipolar plates is also subject to degradation. Park et al. showed that carbon corrosion leads mainly to Pt detachment and/or Pt coarsening by weakened Pt-support interaction, which results in losses in the Pt surface area and the degradation of the performance of the catalyst.[6] After accelerated stress testing, the aggregates of the carbon black support, which are well defined in the original catalyst, were found to be much more reduced and diffused in the cathode catalytic layer. [2] Carbon oxidation and corrosion was also accelerated by the presence of $\mathrm{Pt}$, and it formed oxygen-containing functional groups, such as $\mathrm{C}-\mathrm{O}$ and $\mathrm{C}=\mathrm{O}$, on the surface of the carbon support as confirmed by XPS [11]. The formation of carbon oxides on the surface of the carbon makes the catalyst layer more hydrophilic and leads to the formation of a thin water film on the surface of carbon, resulting in enhanced proton conductivity within the catalyst layer and decreased mass transport due to possible water flooding of hydrophilic pores. The loss of carbon due to complete oxidation of carbon to form $\mathrm{CO}_{2}$ may lead to the collapse of the structure of the CLs and a loss of porosity, increasing the mass transport resistance in the CL.[6] Carbon oxidation may cause the crumbling of the carbon catalystsupport and an increase of its roughness together with the loss of platinum. Catalyst nanoparticles can get trapped in the micro-pores and become not accessible to reactants/Nafion @ resulting is a decrease of catalytic activity.[12] A higher graphitization degree of the carbon support leads to an increase in corrosion resistance, but at the same time can reduce the dispersion of platinum on carbon supports. [13] The functionalization of the graphitic carbon was shown to enhance the distribution of Pt nanoparticles and reduce their agglomeration, resulting in higher stability of Pt catalysts with enhanced activity. [14, 15]

III. Nafion ${ }^{\circledR}$ degradation. Avasarala et al reported the first direct evidence of chemical degradation of Nafion ${ }^{\circledR}$ pendant groups at high cell potential. [5] Prior to that work, the degradation of the main chain through an "unzipping" mechanism was suggested. This 
reaction could lead to the generation within the membrane of peroxide radicals that attack the Nafion ${ }^{\circledR}$ polymer, both at the chain ends, and at the functional.[16] Cationic pollution can detrimentally affect the properties of ionomer. [4] A recent XPS analysis of Nafion®112 after ex situ aging in Fenton solution detected loss of fluorine and sulfur from the membrane and the formation of oxygen-rich moieties in the membrane. [6]

In the pursuit of developing the micro-structural mitigation strategies for PEM Fuel Cells, we have been involved in extensive characterization work of carbon supports and electrocatalysts of different types. [17-19] The performance and corrosion stability of Pt electrocatalysts on morphologically and chemically different carbon supports were investigated in order to understand the effect the support material has on catalyst degradation. Low surface area (LSA), mid-range surface area (MSA), high surface area (HSA), and heat-treated, high surface area (HSA HT) carbons were extensively studied and characterized. [19] We have subjected carbon supports and supported Pt catalysts to accelerated stress testing to monitor the performance losses. Electrode Impedance Spectroscopy (EIS) and Cyclic Voltammetry were used to examine cathode catalyst layer changes. The materials were also characterized using X-Ray Photoelectron Spectroscopy (XPS) and scanning electron microscopy (SEM). [19] The cathode layer thickness, ECSA loss, and subsequently the kinetic loss were found to be least affected for low surface area carbon supports and showed substantially higher degradation for high surface area carbons. HSA carbons resulted in catalysts with smallest relative amounts of metallic Pt and largest amounts of Pt monoxide. Samples with lower surface areas revealed an overall higher content of Pt and $\mathrm{O}$ and Pt dioxide, and this was correlated with high roughness. High surface area samples had the highest percentages of Pt monoxide and the highest amount of smallest pores and were associated with homogeneous morphologies. Heattreated catalyst samples had the highest percentages of metallic Pt and overall carbon, and the highest amount of larger pores.

The changes that carbon-supported platinum electrocatalysts undergo in PEMFC environment were also simulated by ex situ heat treatment of catalyst powder samples at $150^{\circ} \mathrm{C}$ and $100 \% \mathrm{RH}$. In order to study modifications introduced to chemistry, morphology and performance of the electrocatalysts, XPS, HREELS and 3-electrode RDE experiments were performed.[17] Before heat treatment, the graphitic content varied by $20 \%$ among 
samples with different type of carbon support, with distinct differences between bulk and surface compositions within each sample. Following the aging protocol, the bulk and surface chemistry of the samples became similar, with the graphite content increasing or remaining constant, and Pt coordinated to carbon and oxygen decreasing for all samples. From the correlation of changes in chemical composition and losses in performance of the electrocatalysts, we concluded that distribution of Pt particles on graphitic and amorphous carbon is as crucial for electrocatalytic activity as the absolute amount of graphitic carbon present. This was manifested by the largest loss of Pt for amorphous carbon support catalysts.[17]

Thus, X-ray photoelectron spectroscopy, provided a better understanding of the relationships existing between structural changes and carbon surface oxides coverage is emerging.[20]

SEM on thin cross-sections of MEAs provides information on the distribution of the catalyst on the anode and cathode sides, loss of carbon support due to oxidation and deposition of metal in the membrane. [21,22] Image processing of microscopic images is a very useful tool for extracting quantitative information about the structure of the investigated objects in images and could be used to establish a link between this information and various non-visual properties of the objects. [23]

The objective of the current study was to characterize physical and chemical changes of aged catalysts coated membranes (CCMs) and to correlate these changes with MEA performance losses and to derive a set of structural metrics of the catalyst layer components, such as support, catalyst, ionomer, etc., which can be used as measures or predictors of durability. CCMs were tested using AST protocols in order to rapidly degrade the cathode catalyst. Conditioned and aged catalysts coated membranes were then analyzed using XPS to quantify chemical structural changes, and SEM to quantify morphological changes. XPS is a powerful technique to study the chemical changes in the catalysts layers and polymer membrane. XPS work on membrane-electrode assemblies (MEAs) has been reported in [9, 10]. Quantitative elemental analysis carried out by XPS measurements of a fragment from the MEA disclosed changes in $\mathrm{C}$ and $\mathrm{O}$ content and, in particular, highlighted the decrease of Pt content in the catalyst. It is critically important to find design descriptors of the carbon support itself as an indication of degradation behavior 
of electrocatalysts. Table 1 shows the descriptors that have been obtained in preliminary work by our group for carbon supports and catalyst powders and structural and performance characteristics obtained in the current study.

\section{Experimental details}

2.1. Materials. In order to understand the effect of the properties of carbon support on catalyst degradation, Pt catalysts on different carbon supports, low surface area (LSA), midrange surface area (MSA), two different high surface area (HSA) carbons and, in addition, heat treated high HSA surface area carbon (HSAHT) Pt catalyst, each with $50 \mathrm{wt} \% \mathrm{Pt}$, were investigated. The surface area of the carbon supports ranged from less than $200 \mathrm{~m}^{2} \mathrm{~g}^{-1}$ to $800 \mathrm{~m}^{2} \mathrm{~g}^{-1}$. Catalyst coated membranes (CCM) were prepared in-house using the following cathode catalysts with $0.4 \mathrm{mg} \mathrm{Pt}^{-2}$ Pt loading: Pt50LSAC, Pt50MSAC, Pt50HSAC1, Pt50HSAC1-HT, Pt50HSAC2, and Pt50HSAC2-HT. The anode catalyst was Pt50LSAC at a loading of $0.1 \mathrm{mg} \mathrm{Pt} \mathrm{cm}^{-2}$, the membrane was Nafion® NR-211, and the GDL was obtained from AvCarb. The catalyst to Pt ratio was kept constant resulting in catalyst layers thicknesses between 8 and $30 \mu \mathrm{m}$ depending on the support surface area.

2.2 MEA Testing. MEAs were operated at $80^{\circ} \mathrm{C}, 100 \% \mathrm{RH}$ with 5 psig back pressure of hydrogen and air on the anode and cathode sides, respectively. The cells were run at constant flow rates with anode and cathode stoichiometric ratios of $>10$ stoich at $1 \mathrm{~A} \mathrm{~cm}^{-2}$. The testing was carried out with synthesized air to ensure that air contamination did not convolute the results. Furthermore, after testing the membrane thickness was determined in all cases to ensure that the membrane did not influence the findings. The beginning of test (BOT) performance of the MEA was obtained after the MEAs were conditioned overnight. The MEAs were subsequently subjected to AST cycling using a square wave profile with a lower potential limit (UPL) of $0.6 \mathrm{~V}$ vs. anode potential (RHE) for 30s and upper potential limit (UPL) of $1.2 \mathrm{~V}$ vs. anode potential (RHE) for 60s. MEAs using Pt50LSAC as the cathode were also subjected to additional cycling protocols in which the upper potential limit (UPL) was set to $1.0,1.3,1.4$ or $1.6 \mathrm{~V}$ vs. anode potential (RHE). For UPL values lower than $1.3 \mathrm{~V}, 4700$ cycles were done, for higher UPL values smaller number of cycles were utilized as shown in Table 2. 
2.3. XPS Analysis. Conditioned and aged CCMs were analyzed using a Kratos Axis DLD Ultra spectrometer using monochromatic $\mathrm{Al} \mathrm{K \alpha} \mathrm{X}$-ray source, with emission voltage of 15 $\mathrm{kV}$ and emission current of $10 \mathrm{~mA}$. High resolution spectra for carbon, fluorine, oxygen, and platinum were acquired to determine to what extent chemical shifts had altered the carbon support, platinum nanoparticles or Nafion ${ }^{\circledR}$ ionomer. No charge compensation was necessary, resulting in unambiguous peak positions of spectra for accurate peak identifications. High resolution $\mathrm{C}$ and $\mathrm{Pt}$ spectra were curve-fit using individual symmetrical peaks to develop speciation information. The peak width was constrained to that from PTFE standard between 1.0-1.2 eV.

2.4. SEM and Digital Image Processing. SEM images were acquired for conditioned and degraded CCMs. The SEM used in this research work is a Hitachi S-5200 Nano SEM. The images were taken at $2.5 \mathrm{~K}$ and $50 \mathrm{~K}$ magnification at a voltage of $1 \mathrm{kV}$. Resolution is $2 \mathrm{~nm}$ at $1 \mathrm{kV}$. Multiple locations were chosen per sample, and the locations were chosen randomly in order to prevent user bias from influencing the results. The images were analyzed with Digital Image Processing software to quantify changes in sample morphology due to conditioning and accelerated stress testing.[17, 24-27]

\section{Results and discussion}

\subsection{Results of AST.}

Figure 1a shows the kinetic and non-kinetic (Ohmic and mass transport) voltage losses as a function of overall MEA performance. The voltage break-down technique that was used has been described in detail by A. Young et al. [28] The kinetic voltage loss is obtained by fitting the oxygen and air polarization curves to the Tafel equation; corrected for $\mathrm{H}_{2}$ crossover and $\mathrm{iR}$. While the overall MEA performance is predominantly affected by kinetic voltage loss, for degraded MEAs the non-kinetic voltage loss can become substantial. Both voltage loss mechanisms show a transition in slope at $\sim 650 \mathrm{mV}$ separating a range of "normal" degradation and severe corrosion. At the transition point, the non-kinetic voltage loss increases rapidly with decreasing MEA performance due to corrosion of the catalyst layer which is associated with a catalyst layer thinning i.e. carbon loss and losses in porosity, diffusivity and electronic percolation. As expected, the kinetic voltage loss is 
much less affected; it is predominantly due to Pt dissolution and thus a change in ECSA. Figure $1 \mathrm{~b}$ reveals a linear relationship between the ionic resistivity of the catalyst layer and the overall MEA performance indicating that the non-kinetic voltage loss shown in Figure $1 \mathrm{a}$ is at least in part due to a change in the catalyst layer ionic resistance.

Table 2 shows the voltage loss as a function of AST UPL and carbon type. For low surface area carbon, the losses are much smaller than for HSA carbon samples; however, at higher upper potential limits the losses increase substantially. Even at very small number of cycles for 1.6 V UPL, the losses are much higher than at $1.2 \mathrm{~V}$ UPL. High surface area carbon 2 shows the onset of corrosion at UPLs higher than $1.0 \mathrm{~V}$, and losses of $360 \mathrm{mV}$ at 1.2 V. Heat treatment of catalysts on this catalyst does not improve its durability while the heat treated HSAC1 HT catalyst sample shows losses identical to LSAC sample, indicating that heat treatment improves the durability of this catalyst significantly.

We will refer to the samples in two categories: (1) aged after AST: those that did not show performance losses or visual evidence or of degradation and (2) corroded after AST: those that had performance losses and visual changes of CCM appearance.

\subsection{Surface chemistry-to-performance correlations.}

XPS provides a chemical composition from top 5-10 nm of the material. . Six peaks in the spectra come from the catalyst itself with a major component identified as graphitic carbon (Cgr, $284.3 \mathrm{eV}$ ), aliphatic carbon (C-C, $285 \mathrm{eV}$ ), secondary carbons (C*-C-0, 286.0 $\mathrm{eV}$ ) and three types of carbon-oxygen bonding (C-OC/C-OH, $286.9 \mathrm{eV} ; \mathrm{C}=0,288 \mathrm{eV}$ and $\mathrm{COOH}, 289.3 \mathrm{eV}$ ). Ionomer contributes mainly to the higher binding energy regions with three peaks identified as partially fluorinated carbons (C-F, $290.2 \mathrm{eV}$ ), fully fluorinated carbons $\left(\mathrm{CF}_{2}-\mathrm{CF}_{2}, 291 \mathrm{eV}\right)$ and peak due to the $\mathrm{C}-\mathrm{F}_{3}$ end-group or $\mathrm{C}-\mathrm{OF}_{2}$ groups in the polymer pendant side-chain $(292.9 \mathrm{eV})$. Quantitative results of the distribution of different types of carbon species provided by XPS are useful as they are representative of oxidation changes in the carbon chain (amount of $\mathrm{C}_{\mathrm{x}} \mathrm{O}_{\mathrm{y}}$ species) as well as a measure of ionomer morphology due to the ability to separate C-F (located in side chain and branch point) and $\mathrm{CF}_{2}$ (mainly located in the backbone). Molecular dynamics and experimental studies of ionomers films coated on graphite and Pt/graphite surfaces have shown that the Nafion ${ }^{\circledR}$ 
morphology is dependent on the surface characteristics of the substrate. [13-15] For example, Mashio et al [13], have shown through molecular dynamics studies of Nafion ${ }^{\circledR}$ oligomers on bare graphite that the ionomer is predominantly adsorbed on the graphitized carbon sheet via the backbone, while the side chains face toward the pore space. The addition of ionized functional groups to the graphite surface resulted in an increase in the number of sulfonic acid groups near the graphite surface. Lamas et al [14], molecular dynamic studies of the polymer-catalyst-carbon interface have shown that the side chains interact preferentially with the $\mathrm{Pt}$ catalytic particles through the sulfonic groups. Therefore, depending on the surface characteristics of the catalyst, the ionomer morphology may have a varying degree of backbone or side chain interacting with the catalyst and facing pore space as shown schematically (16) in Figure 2. The ratio of C-F to $\mathrm{CF}_{2}$ peaks, which are clearly separated in the $\mathrm{C} 1 \mathrm{~s}$ XPS spectrum, is a useful measure of ionomer distribution and interaction with carbon support. For Nafion®1100 EW, the theoretical ratio of $\mathrm{CF}_{2} / \mathrm{CF}=9$. Results with values greater than 9 would suggest the surface of the pore is more backbone rich and less hydrophilic, while values less than 9 suggest a more side chain rich, hydrophilic surface.

Figure 3 shows high resolution C 1s XPS spectra for LSAC and HSAC samples after the same AST protocol of $1.2 \mathrm{~V}$ of UPL. In corroded HSAC $1.2 \mathrm{~V} \mathrm{CCM}$, there is an obvious increase in peaks at high binding energy part of the spectra due to $C_{x} O_{y}(286-289 e V), C-F$ and $\mathrm{O}-\mathrm{CF}_{2} / \mathrm{CF}_{3}(292 \mathrm{eV})$. Upon separating the CCM from the GDL for some corroded samples, pieces of GDL stuck to the CCM. It is, however, necessary to separate the catalyst support degradation changes occurring during AST from the possible contribution to the $\mathrm{C}$ spectrum from physically present pieces of GDL.

Figure 4 displays $\mathrm{C}$ photoelectron images for corroded HSAC 1 sample, showing the spatial distribution of CL-CCM (Figure 4a) and GDL (Figure 4c). The dark areas in Figure 4a and bright area in Figure 4c are due to the pieces of GDL present on top of the CCM. 110 micron spectra acquired from areas chosen on these images (marked as circles in Figure 4a and 4c) are from CCM in Figure 4b and from a piece of GDL in Figure 4d. The peak at a high binding energy above $292 \mathrm{eV}$ is only present in the spectrum obtained from the GDL piece. Reference C 1s spectra from untested CCM and GDL were also acquired, and Figures 4e and 4f show a high binding energy region of these spectra. Indeed, there is a peak at the binding 
energy of $\sim 292 \mathrm{eV}$ in the spectrum of GDL. This peak is also larger in the corroded sample (Figure 4b). Thus, due to physical attachment of GDL to the CCM upon separation, peak at $292 \mathrm{eV}$ cannot be used as a measure of changes caused by AST cycling itself. Other peaks that form during AST (between 287 and $291.1 \mathrm{eV}$ ) are not affected by the GDL presence as confirmed by the reference GDL spectrum. Consequently, the peaks at binding energies higher than $292 \mathrm{eV}$ were excluded from the quantitative results discussed below.

As hypothesized above, the Nafion ${ }^{\circledR}$ morphology in the catalyst layer indeed depends on the surface chemistry of the catalyst powder, particularly the amount of surface functional groups which can be expressed as oxygen concentration ( 0 at \%) and amount of secondary carbons $\left(\mathrm{C}^{*}-\mathrm{C}_{\mathrm{x}} \mathrm{O}_{\mathrm{y}}\right)$ detected in catalyst powders themselves. Figure 5 a shows that absolute amounts of both $\mathrm{CF}$ and $\mathrm{CF}_{2}$ in catalysts layers are larger for catalysts that have a higher degree of surface oxides as expressed by the oxygen present in the catalysts.

Conditioning is an important step during which the ionomer swells and its morphology changes. In the conditioned catalyst layer, the ratio of backbone species $\left(\mathrm{CF}_{2}\right)$ to that of $\mathrm{CF}$, which depends on ionomer distribution and interaction with carbon support, is smaller for samples with higher functionalization as shown in Figure $5 \mathrm{~b}$ which plots the $\mathrm{CF}_{2} / \mathrm{CF}$ ratio for conditioned catalyst layers as a function of the amount of secondary carbons in the catalyst powders. As the carbon support becomes more functionalized, the larger amount of C-F groups (side chains) face the pore resulting in weaker interaction of ionomer with carbon support.

The ratio of side chains $\mathrm{CF}$ to backbone $\mathrm{CF}_{2}$ increases upon humidification for all types of carbon support, as is shown in Figure 6. This suggests that ionomer undergoes a rearrangement so that the hydrophilic side chains interact with the water in humidified pore. Annealing studies of ultrathin Nafion ${ }^{\circledR}$ films by Paul et al. [17] have shown that the ionomer undergoes a surface and bulk rearrangement that results in a simultaneous increase in surface hydrophobicity and drop of conductivity.

XPS provides critical information on the changes in the chemical composition of the electrocatalyst within the catalyst layer as a function of AST UPL. Significant changes due to carbon corrosion can be expressed by the degree of oxidation, measured as the total amount of carbon-oxygen species, $\mathrm{C}_{\mathrm{x}} \mathrm{O}_{\mathrm{y}}$ (which are equal to the total amounts of $\mathrm{C}-\mathrm{O}, \mathrm{C}=\mathrm{O}$ and $\mathrm{COOH}$ species in $\mathrm{C} 1 \mathrm{~s}$ spectra). Changes in ionomer morphology can be expressed as 
relative amounts of $\mathrm{CF}_{2}$ and $\mathrm{CF}$ species. This ratio is higher for more stable ionomer when it interacts with the surface functional groups of the carbon support. Upon carbon support oxidation, the ionomer rearranges so that hydrophilic C-F groups face the pore. The smaller the degree of oxidation, the higher degree of ionomer interaction with the carbon support, the higher the ECSA retained during AST cycling. The higher the amount of functionalized groups on the surface of electrocatalysts, the more of C-F side chains face the pore and the weaker the interaction of ionomer with the carbon support.

In addition to carbon chemistry, Pt speciation is derived from Pt $4 \mathrm{f}$ high resolution spectra deconvoluted into two chemical types (not shown). The low binding energy value of $71.4 \mathrm{eV}$ for the $4 \mathrm{f} 7 / 2$ peak is assigned to metallic platinum while the higher one at 72.7 $\mathrm{eV}$ is assigned to the mixture of platinum chemically coordinated with the carbon support and the oxidized platinum (Pt-C+Pt-0). Table 3 shows amounts of carbon and platinum species after MEAs were subjected to different AST protocols in comparison with the initial amounts present in conditioned MEAs.

For LSAC, the amounts of surface oxides $\mathrm{C}_{\mathrm{x}} \mathrm{O}_{\mathrm{y}}$ and ionomer side chains $\mathrm{C}-\mathrm{F}$ increase with higher UPL of AST. Oxidation of carbon during AST is, thus, accompanied with ionomer exposing its hydrophilic side chains. This is associated with a decrease in the amount of side chains of ionomer, $\mathrm{CF}_{2}$, which occurs even at low AST potentials. The change in the $\mathrm{Pt}$ environment is also obvious; the amount of metallic Pt increases at the expense of a decrease in the amount of Pt that is coordinated with carbon and oxidized Pt. The increase in the relative amount of metallic Pt is correlated with the growth of Pt size on the cathode. LSAC is stable between 1.0 and $1.2 \mathrm{~V}$, and, at higher potentials, the changes in its structure become more dramatic. The change in the chemical environment for HSAC samples is greater than for LSAC even at lower potentials. The oxidation of high surface area carbon is prominent at an UPL of $1.2 \mathrm{~V}$. The associated loss of Pt-C/Pt-O is also much higher at these low potentials for HSAC samples than for LSAC. Heat-treating this sample is not effective as the same change in chemistry during AST protocols is observed for HSAC2 HT samples as for HSAC 2 sample itself. The heat treated HSAC 1 HT sample, however, shows improved stability with respect to HSAC 1 sample, which is reflected by smaller rearrangement of ionomer, the lower degree of carbon oxidation and lower Pt loss. 
Figure 7 shows changes in chemistry of the CCM as detected by XPS as a function of MEA performance metrics, i.e. loss in cell voltage at a current density of $0.3 \mathrm{~A} \mathrm{~cm}^{-2}$, loss in ionic conductivity and electrochemically accessible surface area. The losses in the performance and ionic conductivity are strongly correlated with the amount of oxidized carbon and the amount of exposed side chains C-F species. The ECSA retained after AST is higher for the samples that have the highest amount of graphitic carbon, oxidized Pt and Pt coordinated with C. Carbon oxide species that form on the carbon surface due to corrosion, make the surface more hydrophilic which consequently causes the catalyst layer to be prone to flooding, resulting in decreased diffusion and MEA voltage loss.[12] Despite surface oxidation, graphitic carbon remains unaffected, indicating that it is the amorphous carbon that is susceptible to substantial corrosion. The changes that are occurring with carbon and $\mathrm{Pt}$, suggest that the main loss of platinum is associated with $\mathrm{Pt}$ that is coordinated to amorphous carbon, which readily oxidizes with aging. The Pt that is coordinated to graphitic carbon sites stays intact, and this explains the higher relative amount of metallic Pt found after AST. As was reported previously, TEM analysis has shown two groups of Pt particles, particles in contact with carbon support and particles removed from the carbon support. There are also reports of the formation of Pt0. [16] In our previous durability studies of catalysts powders [17] and this work, we do not see the formation of PtO after AST. For the most corroded samples, our observations confirm the removal of Pt particles from the oxidized amorphous carbon and growth of Pt particles with higher amounts of reduced metallic Pt.

Importantly, we were able to identify the descriptors of the carbon support itself that are indicative of the corrosion behavior that can be used for the design of stable electrocatalysts. Figure 8 shows the BOT performance as well as the performance and ESCA losses with AST cycling as a function of initial graphitic carbon content in the carbon support of different catalyst powders. It reveals that the ECSA loss and performance losses are directly related to the initial graphitic content of carbon. A targeted performance loss of $\leq 15 \%$ after 4700 AST cycles at a UPL of $1.2 \mathrm{~V}$ is satisfied when carbon contains more than $55 \%$ graphitic carbon surface species. The amount of surface oxides in the carbon support that satisfy the $15 \%$ performance loss must be less than $16 \%$.

\subsection{Morphology to performance correlations.}


Figure 9 shows SEM images for conditioned, aged and corroded CCMs at two different magnifications. Careful consideration was taken to ensure that the magnification, voltage, brightness, and contrast were at constant values for all images. From Figure 9, it is clearly visible that the structure of the catalyst layer has changed drastically following the cycling protocol. The corroded sample no longer shows individual carbon black particles that are clearly visible in the conditioned CCM image.

In order to understand impacts of AST on porosity of CCMs, digital image processing of SEM images was performed. [23-25] We focused on skewness $\left(R_{s k}\right)$ as it describes asymmetry of the roughness, pointing to the domination of pores or peaks in the image. Its value increases as the amount of pores (dark values of intensity within images) increases. In addition, we have applied high-pass filter to remove low-frequency component, and lowpass filter to remove high-frequency component from images to produce roughness and waviness image components, respectively. [24] At 2.5K magnification, high-frequency component images correspond to roughness in the range of 0.4-1 $\mu \mathrm{m}$, and low-frequency component images correspond to $5-20 \mu \mathrm{m}$. At $50 \mathrm{~K}$ magnification, filtering separates images into low-frequency components at 0.2-1 micron scale and a high-frequency component at 20-100 nm.

Figure 10 shows skewness parameters extracted from image components separated by filtering. Overall skewness and skewness at scale of 5-20 microns does not change significantly with increasing AST potential. Dramatic increase in porosity, as represented by skewness at scales of 0.2-1 microns and 20-100 nm, is detected as the AST UPL increases. In comparison with conditioned samples, the porosity at a larger scale of 5-20 microns increases for all tested samples. Degradation of MEA, however, is linked mainly to changes at scale from tens of nanometer up to a micron. Such micro roughness and micro porosity play a significant role in water management, potentially, causing failure of MEAs at higher UPL during AST cycling. These observations are in close correspondence with a recent report that showed that the carbon corrosion AST resulted in more porous CLs with larger pores and larger pore volume.[6] In addition, the authors reported the enhancement of pores in the same range of 40-100 $\mathrm{nm}$ as observed in the current work due to the loss of carbon. The degradation of the carbon catalyst-support leads to the evolution of the microstructure. 
The increase in overall porosity is correlated with catalyst layer thickness loss as shown in Figure 11a. The corrosion of the carbon, as manifested by the formation of surface oxides, is accompanied by the loss of the material and, subsequently, increase in the number of pores in MEA. Previous studies also showed that cathode thickness decreased due to loss of the carbon support for the medium surface area carbons at all UPLs (1.0 to $1.4 \mathrm{~V}$ vs NHE) but only for the most aggressive testing protocol for the low surface area carbon support. [29]

The morphological analysis of carbon supports reveals a direct relationship between the morphological properties of the carbon support and the way electrocatalysts behave in the catalyst layer. Indeed, as Figure 11b shows, the larger amount of micro-pores and a smaller amount of macro-pores in carbon support, results in the CL with lowest performance losses and highest retained ECSA that would meet a durability target of $15 \%$.

\subsection{Design levers and durability window}

Figure 12 shows an illustration of carbon support with types of features that must be present in order to meet durability target as derived in Figure 8 and 11.

In summary, based on thorough structural analysis and developed structure-toproperty correlations, the following observation related to catalyst layer durability have been found:

1. The catalyst layer thickness loss is due to carbon corrosion (i.e. carbon loss). Carbon corrosion is characterized by a 2-step mechanism, i.e. carbon oxidation and carbon loss. During the initial oxidation step, oxygen-containing functional groups are formed on the carbon surface. The metric of the degree degradation is the amount of CxOy species as determined by the C 1s XPS spectra

2. The ionomer undergoes a rearrangement with cycling; more C-F groups (side chains) are exposed to the pore (rather than carbon support). This serves as an indication of the interaction of ionomer with the catalyst carbon support. The metric of rearrangement is amounts of $C-F_{2}$ and $C$ - F from $C 1$ S XPS spectra.

3. Carbon corrosion and catalyst layer thickness loss are accompanied by loss of Pt-O and Pt-C and the resulting loss of ECSA. The metric of the Pt loss is the amount of Pt species determined by the Pt $4 f X P S$ spectra. 
4. During AST cycling the catalyst layer micro porosity increases in addition to an increase in overall porosity facilitating proton and water transport. The metric is the skewness parameters determined from SEM images.

5. A graphitic content higher than $55 \%$ and the amount of surface oxides fewer than $16 \%$, as well as a high amount of micro porosity with a lower relative amount of larger pores, and higher relative amount of small 20-100 $\mathrm{nm}$ pores have shown the best performance among the analyzed catalyst layers

\section{Conclusions}

Structure-to-performance correlations have been developed linking catalyst and catalyst layer properties with CCM performance and degradation. The complexity and convolution of the interactions between the different variables of all MEA components need to be considered in MEA optimization. The metrics of chemical and morphological changes derived in this work represent an important tool for MEA designers. Understanding of the relationship between chemical composition and structural properties of the carbon support and durability allows catalyst layer design trade-offs between cost, performance, and durability.

\section{Acknowledgements}

Department of Energy EERE Hydrogen and Fuel Cell Technology Program (Project DEEE0000466). 


\section{References}

[1] USCAR FUEL CELL TECH TEAM CELL COMPONENT ACCELERATED STRESS TEST PROTOCOLS FOR PEM FUEL CELLS in, DOE EERE, 2010.

[2] P. Ferreira-Aparicio, B. Gallardo-Lopez, A.M. Chaparro, L. Daza, Journal of Power Sources, 196 (2011) 4242-4250.

[3] S.J. Bae, S.J. Kim, J.H. Lee, I. Song, N.I. Kim, Y. Seo, K.B. Kim, N. Lee, J.Y. Park, Appl Energ, 131 (2014) 48-55.

[4] L. Dubau, L. Castanheira, F. Maillard, M. Chatenet, O. Lottin, G. Maranzana, J. Dillet, A. Lamibrac, J.-C. Perrin, E. Moukheiber, A. ElKaddouri, G. De Moor, C. Bas, L. Flandin, N. Caqué, Wiley Interdisciplinary Reviews: Energy and Environment, 3 (2014) 540-560.

[5] S.S. Zhang, X.Z. Yuan, H.J. Wang, W. Merida, H. Zhu, J. Shen, S.H. Wu, J.J. Zhang, International Journal of Hydrogen Energy, 34 (2009) 388-404.

[6] J.H. Park, S.D. Yim, T. Kim, S.H. Park, Y.G. Yoon, G.G. Park, T.H. Yang, E.D. Park, Electrochim Acta, 83 (2012) 294-304.

[7] A. Marcu, G. Toth, S. Kundu, L.C. Colmenares, R.J. Behm, Journal of Power Sources, 215 (2012) 266273.

[8] Y.Y. Shao, G.P. Yin, Y.Z. Gao, Journal of Power Sources, 171 (2007) 558-566.

[9] P. Trogadas, T.F. Fuller, P. Strasser, Carbon, 75 (2014) 5-42.

[10] K.J.J. Mayrhofer, M. Arenz, 7 - Catalyst ageing and degradation in polymer electrolyte membrane fuel cells, in: C. Hartnig, C. Roth (Eds.) Polymer Electrolyte Membrane and Direct Methanol Fuel Cell Technology, Woodhead Publishing, 2012, pp. 178-194e.

[11] B. Avasarala, R. Moore, P. Haldar, Electrochim Acta, 55 (2010) 4765-4771.

[12] S. Sharma, B.G. Pollet, Journal of Power Sources, 208 (2012) 96-119.

[13] X.J. Zhao, A. Hayashi, Z. Noda, K. Kimijima, I. Yagi, K. Sasaki, Electrochim Acta, 97 (2013) 33-41.

[14] J.Y. Kim, S. Lee, T.-Y. Kim, C. Pak, H.-T. Kim, Carbon, 77 (2014) 525-537.

[15] J.Y. Kim, S. Lee, T.Y. Kim, H.T. Kim, Electrochim Acta, 134 (2014) 418-425.

[16] J. Peron, Y. Nedellec, D.J. Jones, J. Roziere, Journal of Power Sources, 185 (2008) 1209-1217.

[17] A. Patel, K. Artyushkova, P. Atanassov, V. Colbow, M. Dutta, D. Harvey, S. Wessel, J Vac Sci Technol A, 30 (2012).

[18] A. Patel, K. Artyushkova, P. Atanassov, D. Harvey, M. Dutta, V. Colbow, S. Wessel, Polymer Electrolyte Fuel Cells 11, 41 (2011) 845-852.

[19] A. Patel, K. Artyushkova, P. Atanassov, A. Young, M. Dutta, Z. Ahmad, V. Colbow, S. Wessel, S.Y. Ye, Ecs Transactions, 33 (2010) 425-431.

[20] L. Castanheira, L. Dubau, M. Mermoux, G. Berthomé, N. Caqué, E. Rossinot, M. Chatenet, F. Maillard, ACS Catalysis, 4 (2014) 2258-2267.

[21] P.J. Ferreira, G.J. la O', Y. Shao-Horn, D. Morgan, R. Makharia, S. Kocha, H.A. Gasteiger, Journal of the Electrochemical Society, 152 (2005) A2256-A2271.

[22] R. Srivastava, P. Mani, P. Strasser, Journal of Power Sources, 190 (2009) 40-47.

[23] A. Bigdeli, M.R. Hormozi-Nezhad, M. Jalali-Heravi, M.R. Abedini, F. Sharif-Bakhtiar, Rsc Adv, 4 (2014) 60135-60143.

[24] K. Artyushkova, S. Pylypenko, M. Dowlapalli, P. Atanassov, Rsc Adv, 2 (2012) 4304-4310.

[25] K. Artyushkova, S. Pylypenko, M. Dowlapalli, P. Atanassov, Journal of Power Sources, 214 (2012) 303-313.

[26] T.V. Reshetenko, J. St-Pierre, K. Artyushkova, R. Rocheleau, P. Atanassov, G. Bender, M. Ulsh, Journal of the Electrochemical Society, 160 (2013) F1305-F1315. 
[27] C. Santoro, M. Guilizzoni, J.P.C. Baena, U. Pasaogullari, A. Casalegno, B. Li, S. Babanova, K. Artyushkova, P. Atanassov, Carbon, 67 (2014) 128-139.

[28] A.P. Young, J. Stumper, S. Knights, E. Gyenge, Journal of The Electrochemical Society, 157 (2010) B425-B436.

[29] A.P. Hitchcock, V. Berejnov, V. Lee, M. West, V. Colbow, M. Dutta, S. Wessel, Journal of Power Sources, 266 (2014) 66-78. 


\section{List of Figures}

Figure 1. (a) MEA voltage loss breakdown into kinetic and non-kinetic contributions (b) MEA performance as a function of catalyst layer ionic resistivity

Figure 2. Two possible configurations of ionomer in catalyst layer are displayed (16)

Figure 3. High resolution C 1s spectrum for (a) LSAC and (b) HSAC aged at 1.2 AST UPL.

Figure 4. C 1s image and small area $C$ 1s spectra representing CL-rich region of CCM (a) and (b) and GDLrich region of CCM (c) and (d). Circles on the images indicate the area from which spectra were acquired. High binding energy range of $\mathrm{C} 1 \mathrm{~s}$ spectra is shown for reference CCM (e) and GDL (f).

Figure 5. ((b) $\mathrm{CF}_{2} / \mathrm{CF}$ ratio in the conditioned $\mathrm{CCM}$ as a function of the amount of functionalized carbon in catalyst powder.

Figure 6. Change in $\mathrm{C}-\mathrm{F}$ and $\mathrm{C}-\mathrm{F}_{2}$ amounts in dry (non-run) and conditioned $\mathrm{CCMs.}$

Figure 7. Structure-to-property correlations. \% of C-O and C-F\% as a function of loss in voltage (a) and loss in ionic conductivity (c). \%Cgr and $\mathrm{CF}_{2} / \mathrm{CF}(\mathrm{b})$ and $\mathrm{PtO}+\mathrm{PtC}$ and $\mathrm{CF}_{2}(\mathrm{~d})$ as a function of ESCA retained.

Figure 8. BOT performance, ECSA loss and performance loss in \% as a function of graphitic carbon in carbon support powder. The durability window of $15 \%$ losses is highlighted.

Figure 9. SEM images at two different magnifications for conditioned, aged after AST and corroded after AST HSAC CCM.

Figure 10. Skewness at four different scales as a function of AST UPL.

Figure 11. Morphology-to-performance correlations. Overall skewness of CCMs and amount of surface oxides as a function of thickness loss (a). Cathode ESCA and performance loss in $\mathrm{V}$ as a function of micro skewness (b). The durability window of $15 \%$ losses is highlighted.

Figure 12. Structure of carbon support allowing catalyst layers that meet durability windows of $15 \%$ loss: uniform high micro-porous morphologies with high graphitic content and low amount of surface oxides. 


\section{Tables}

Table 1. Descriptors for carbon supports, catalyst powders, structure and performance characteristics.

\begin{tabular}{|c|c|c|c|c|}
\hline \multirow{2}{*}{\multicolumn{2}{|c|}{$\begin{array}{c}\text { Carbon support } \\
\text { Surface chemistry (XPS): } \\
\text { C/O, Cx-Oy types }[18,19]\end{array}$}} & \multirow{2}{*}{$\begin{array}{c}\text { Catalyst powder } \\
\text { Surface Chemistry (XPS): } \\
\text { Pt\%, C/O, Cx-Oy types, } \\
\text { Pt type[17] }\end{array}$} & \multicolumn{2}{|c|}{$\begin{array}{l}\text { Catalyst Layer and MEA } \\
\text { (current paper) }\end{array}$} \\
\hline & & & $\begin{array}{c}\text { Surface Chemistry (XPS): Pt } \\
\text { loading, } \mathrm{C} / \mathrm{O}, \mathrm{Cx}-\mathrm{Oy} \text { types, } \mathrm{C}- \\
\mathrm{F} / \mathrm{CF}_{2} ; \mathrm{Pt} \text { type }\end{array}$ & Performance \\
\hline \multicolumn{2}{|c|}{ Morphology (SEM)[18, 19] } & $\begin{array}{l}\text { Morphology (SEM): } \\
\text { roughness/porosity[17] }\end{array}$ & $\begin{array}{l}\text { Morphology (SEM): } \\
\text { roughness/porosity }\end{array}$ & $\begin{array}{l}\text { Voltage Loss } \\
\text { Break-down }\end{array}$ \\
\hline $\begin{array}{l}\text { Aggregate } \\
\text { distribution }\end{array}$ & $\begin{array}{l}\text { porosity } \\
\text { /roughness }\end{array}$ & $\begin{array}{l}\text { Pt size distribution } \\
\text { (TEM)[17] }\end{array}$ & CL thickness & $\begin{array}{l}\text { CL Thickness } \\
\text { change }\end{array}$ \\
\hline \multirow{3}{*}{\multicolumn{2}{|c|}{ Surface Area: BET $[18,19]$}} & & Surface area: ECSA & ECSA losses \\
\hline & & & CL ionic resistance & \\
\hline & & & $\mathrm{O}_{2}$ diffusivity & \\
\hline
\end{tabular}

Table 2. MEA performance loss at $0.3 \mathrm{~A} \mathrm{~cm}^{-2}$ as a function of AST UPL.

\begin{tabular}{|c|c|c|}
\hline & $\begin{array}{l}\text { AST UPL, } \\
\text { \# of cycles }\end{array}$ & $\begin{array}{c}\text { Loss in V, } \\
0.3 \mathrm{~A} \mathrm{~cm}^{-2}, \text { Air } \\
(\mathrm{mV})\end{array}$ \\
\hline Pt50LSAC & $1 \mathrm{~V}, 4700$ cycles & 11.5 \\
\hline Pt50LSAC & $1.2 \mathrm{~V}, 4700$ cycles & 24.0 \\
\hline Pt50LSAC & $1.3 \mathrm{~V}, 4700$ cycles & 138.2 \\
\hline Pt50LSAC & $1.4 \mathrm{~V}, 700$ cycles & 161.1 \\
\hline Pt50LSAC & $1.6 \mathrm{~V}, 50$ cycles & 57.3 \\
\hline Pt50HSAC2 & 1V, 4700 cycles & 39.4 \\
\hline Pt50HSAC2 & $1.2 \mathrm{~V}, 4700$ cycles & 361.9 \\
\hline Pt50HSAC1-HT & $1.2 \mathrm{~V}, 4700$ cycles & 21.6 \\
\hline Pt50HSAC2-HT & $1.2 \mathrm{~V}, 4700$ cycles & 357.0 \\
\hline
\end{tabular}


Table 3. Change in speciation of C and Pt as derived from XPS spectra as a function of AST UPL

\begin{tabular}{lcccccc} 
& AST UPL, $\mathrm{V}$ & $\mathrm{C}_{\mathrm{x}} \mathrm{O}_{\mathrm{y}}$ & $\mathrm{C}-\mathrm{F}$ & $\mathrm{CF}_{2}$ & $\mathrm{Pt}$ & Pt-O/Pt-C \\
\hline Pt50LSAC & Conditioned & 5.7 & 2.6 & 31.9 & 56.3 & 43.7 \\
Pt50LSAC & $\mathbf{1 . 0}$ & 6.4 & 3.3 & 19.6 & 57.8 & 42.2 \\
Pt50LSAC & $\mathbf{1 . 2}$ & 9.0 & 5.3 & 23.3 & 60.3 & 39.7 \\
Pt50LSAC & $\mathbf{1 . 3}$ & 28.4 & 21.3 & 14.7 & 62.4 & 37.6 \\
Pt50LSAC & $\mathbf{1 . 4}$ & 35.8 & 25.5 & 14.5 & 62.4 & 37.6 \\
Pt50LSAC & $\mathbf{1 . 6}$ & 34.8 & 27.6 & 14.7 & 58.0 & 42.0 \\
& & & & & & \\
Pt50HSAC2 & Conditioned & 7.4 & 3.5 & 18.1 & 47.0 & 53.0 \\
Pt50HSAC2 & $\mathbf{1 . 0}$ & 5.8 & 2.4 & 14.0 & 53.7 & 46.3 \\
Pt50HSAC2 & $\mathbf{1 . 2}$ & 30.5 & 20.6 & 14.6 & 62.7 & 37.3 \\
& & & & & & \\
Pt50HSAC1-HT & Conditioned & 7.6 & 3.3 & 19.2 & 49.7 & 50.3 \\
Pt50HSAC1-HT & $\mathbf{1 . 2}$ & 9.7 & 5.7 & 25.6 & 59.6 & 40.4 \\
\hline Pt50HSAC2-HT & Conditioned & 6.8 & 2.8 & 18.8 & 53.0 & 47.0 \\
\hline Pt50HSAC2-HT & $\mathbf{1 . 2}$ & 25.4 & 20.1 & 18.9 & 64.4 & 35.6
\end{tabular}


Figures

a)

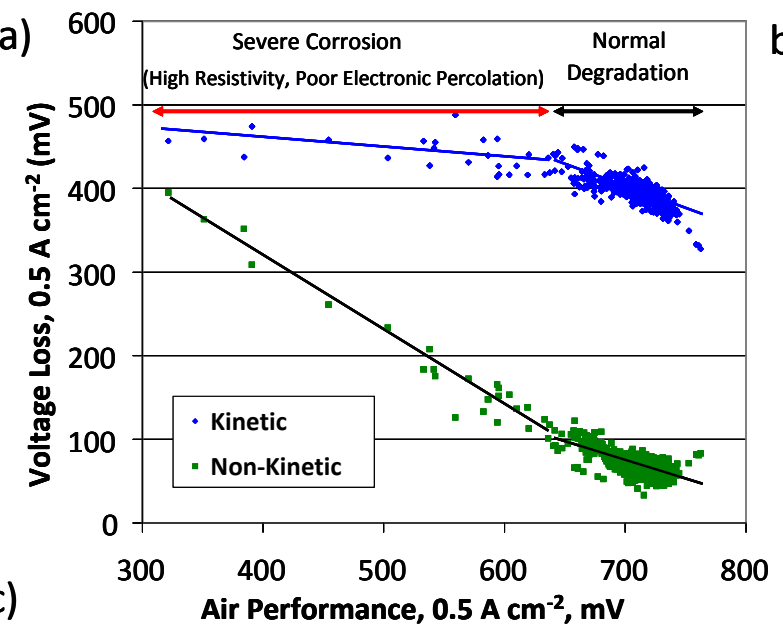

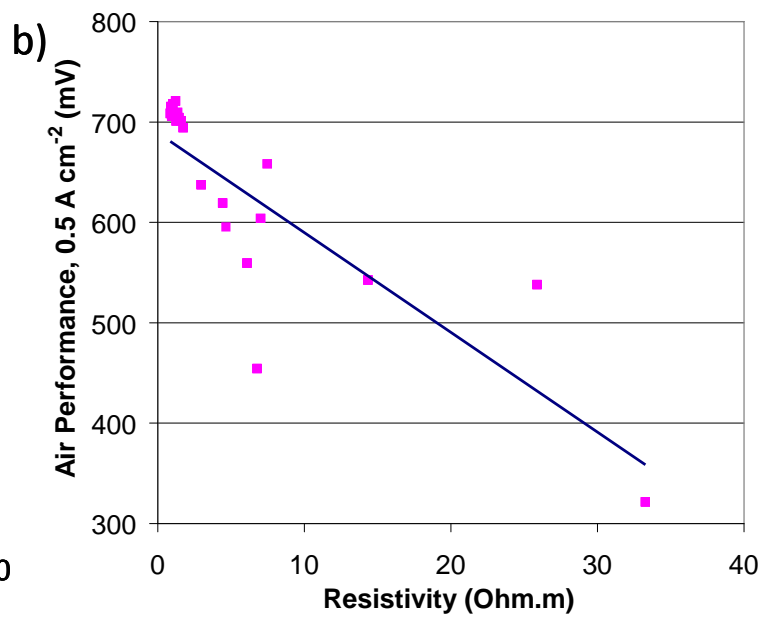

Figure 1. (a) MEA voltage loss breakdown into kinetic and non-kinetic contributions (b) MEA performance as a function of catalyst layer ionic resistivity

\section{Nafion $^{\circledR} 1100 \mathrm{EW}$ $\mathrm{CF}_{2}: \mathrm{CF}=9$}

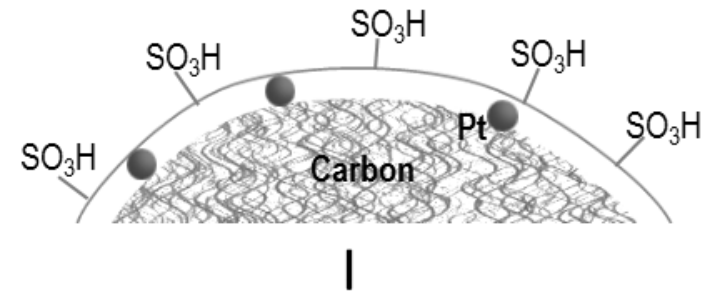

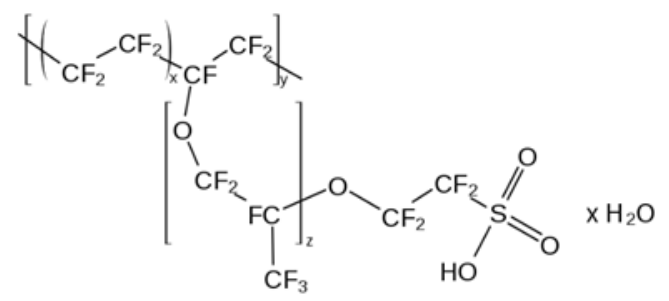

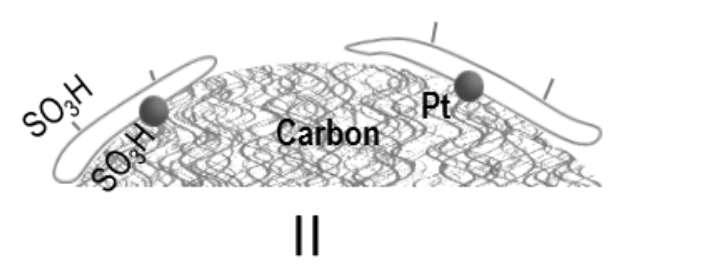

Figure 2. Two possible configurations of ionomer in catalyst layer are displayed (16) 

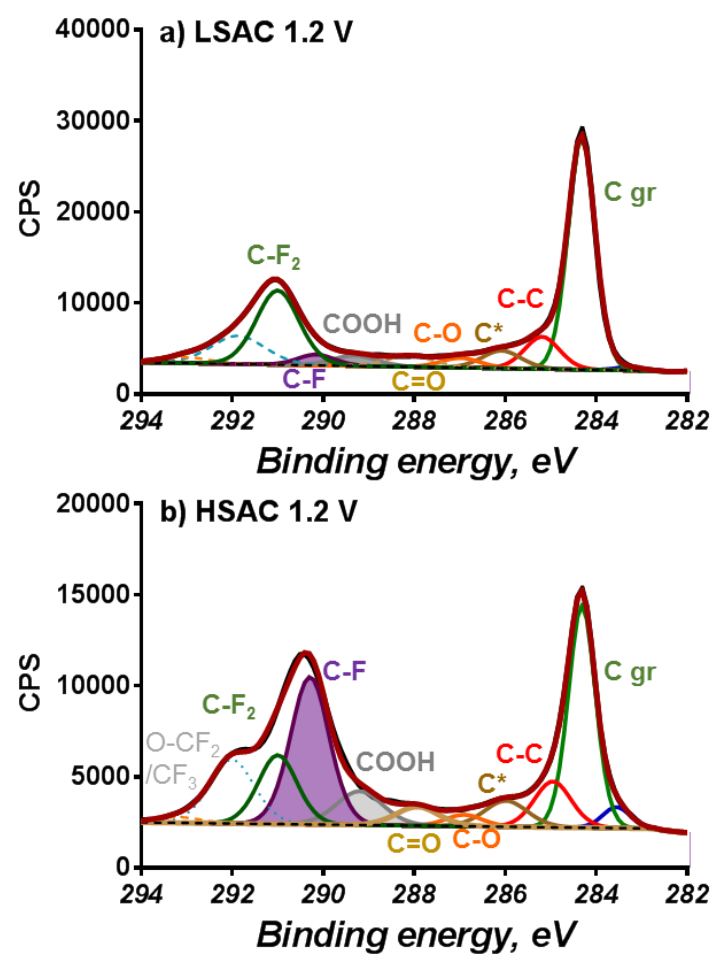

Figure 3. High resolution C 1s spectum for (a) LSAC and (b) HSAC aged at 1.2 AST UPL.

a)

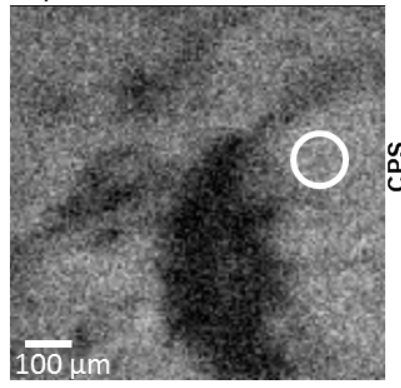

c)

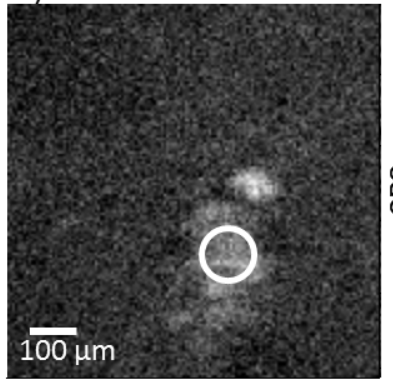

b)

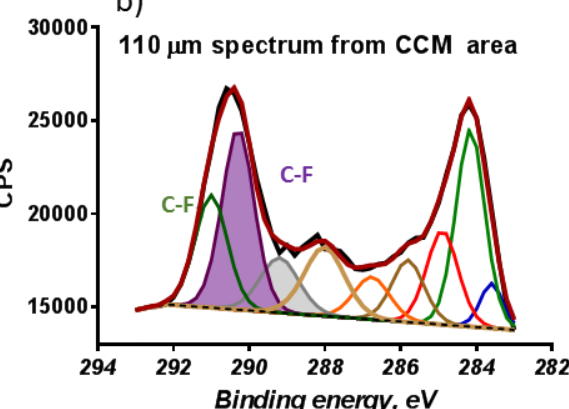

d)

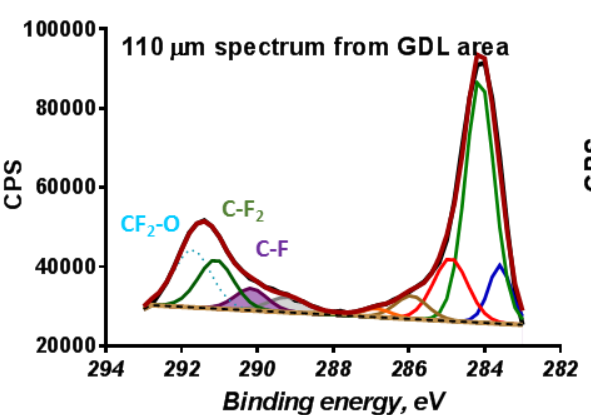

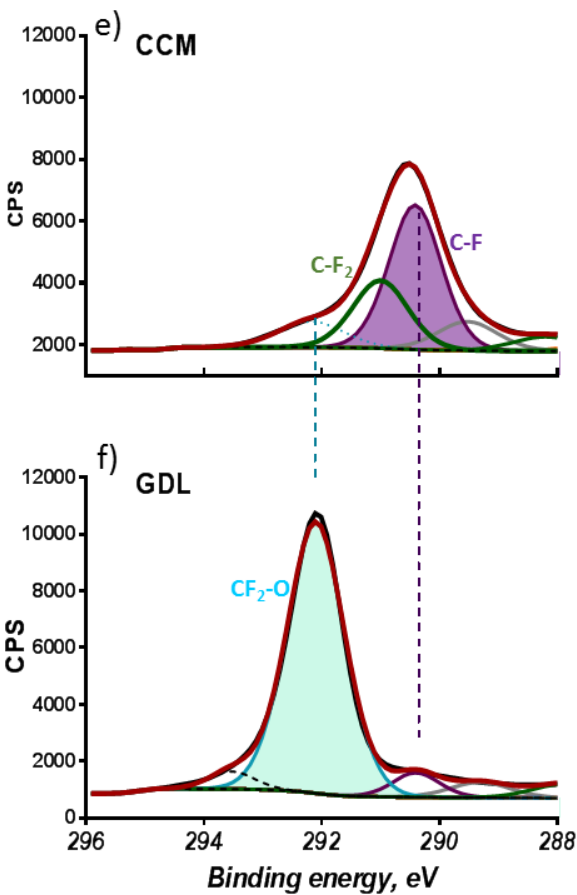

Figure 4. C 1s image and small area C 1s spectra representing CL-rich region of CCM (a) and (b) and GDL-rich region of CCM (c) and (d). Circles on the images indicate the area from which spectra were acquired. High binding energy range of $C 1 \mathrm{~s}$ spectra is shown for reference CCM (e) and $\operatorname{GDL}(f)$. 

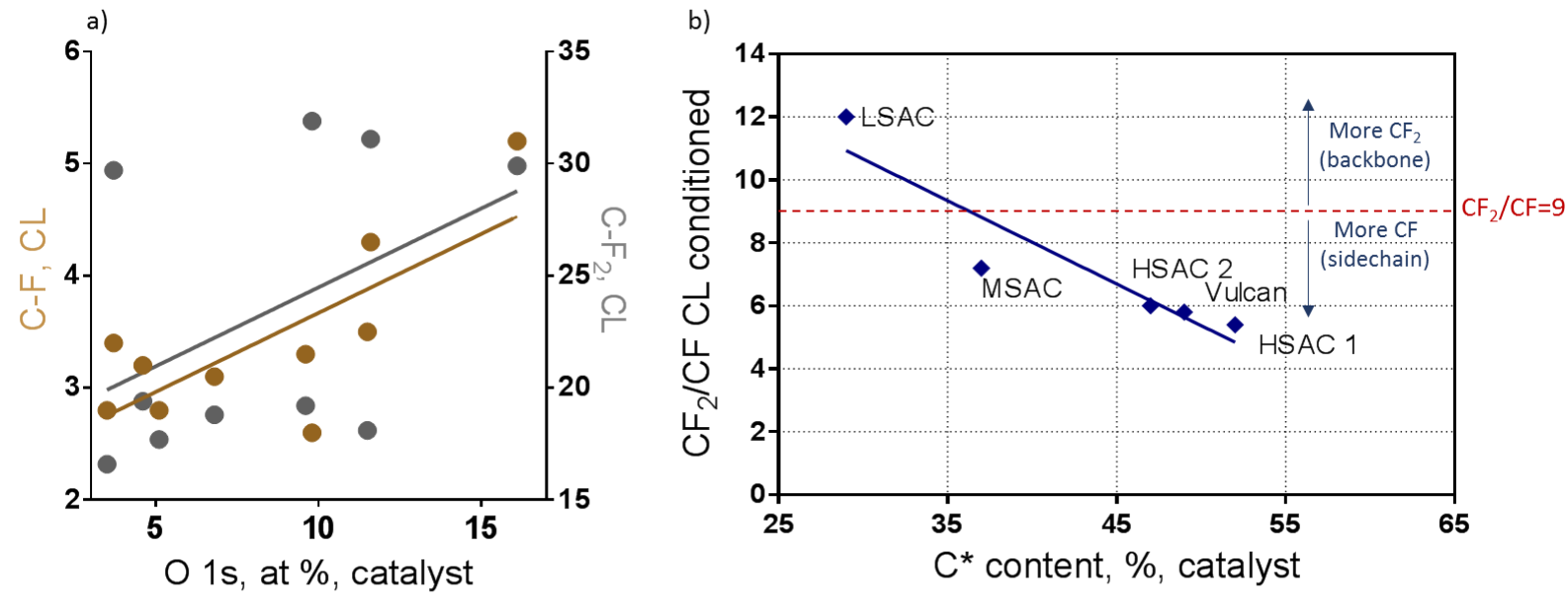

Figure 5. (a) Amount of ionomer in catalyst layer a) Amount of C-F and C-F2 species in the catalyst layer as a function of oxygen at\% in catalyst powder. as a function of oxygen at\% in catalyst powder. (b) $\mathrm{CF}_{2} / \mathrm{CF}$ ratio in the conditioned CCM as a function of the amount of functionalized carbon in catalyst powder.
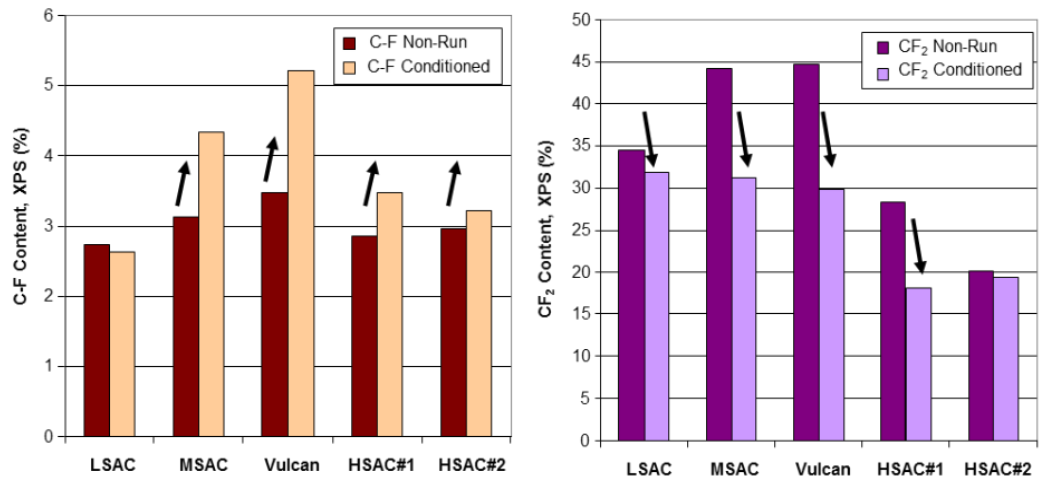

Figure 6. Change in C-F and C-F2 amounts in dry (non-run) and conditioned CCMS. 

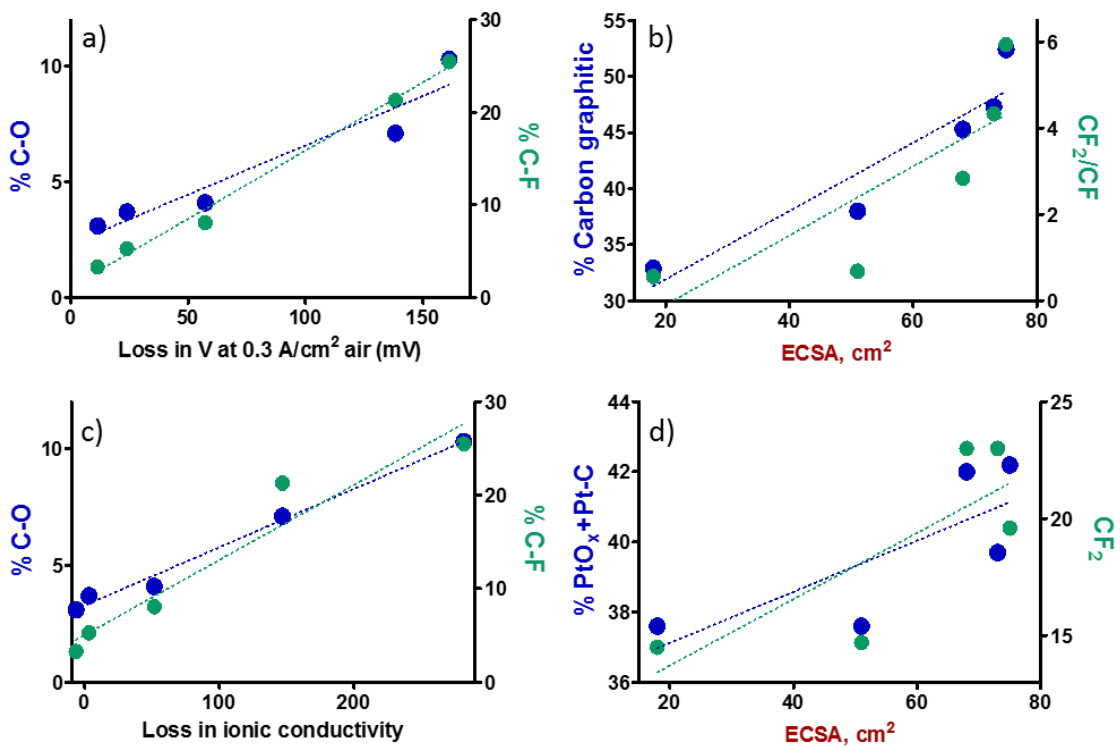

Figure 7. Structure-to-property correlations. \% of C-O and C-F\% as a function of loss in voltage (a) and loss in ionic conductivity (c). \% Cgr and $\mathrm{CF}_{2} / \mathrm{CF}(\mathrm{b})$ and $\mathrm{PtO}+\mathrm{PtC}$ and $\mathrm{CF}_{2}(\mathrm{~d})$ as a function of ESCA retained.

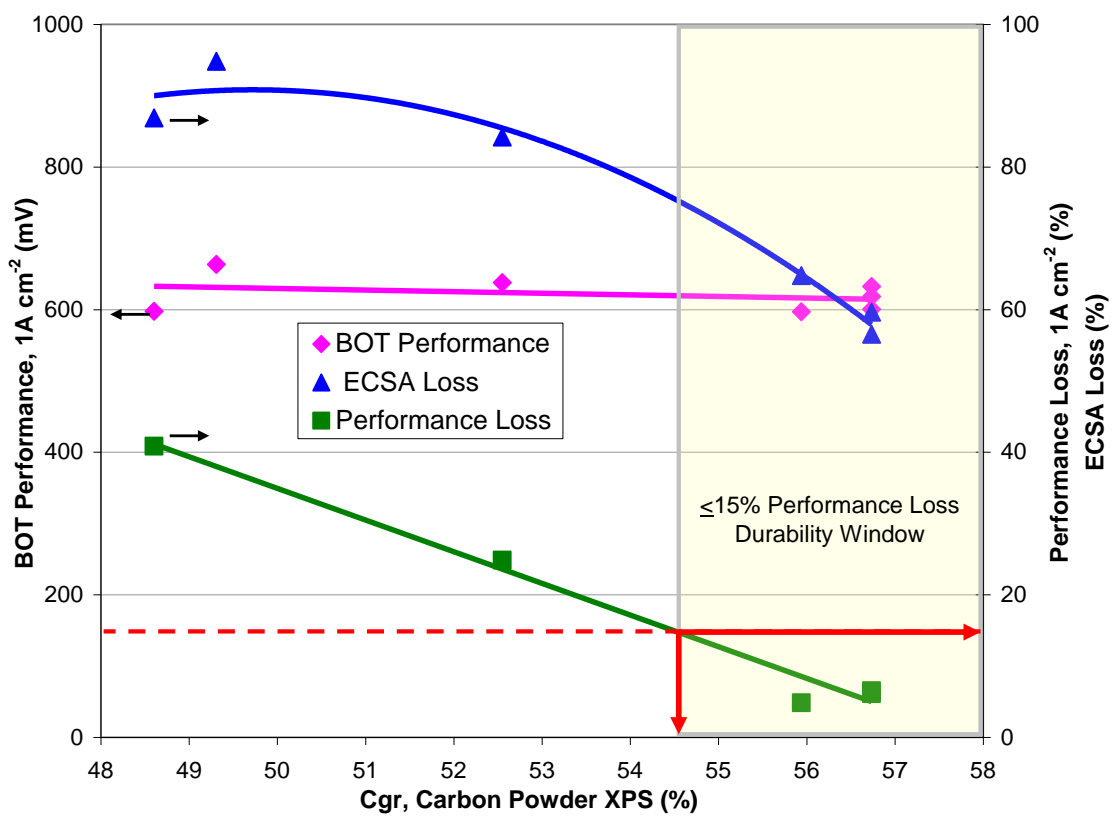

Figure 8. BOT performance, ECSA loss and performance loss in \% as a function of graphitic carbon in carbon support powder. The durability window of $15 \%$ losses is highlighted. 
Conditioned
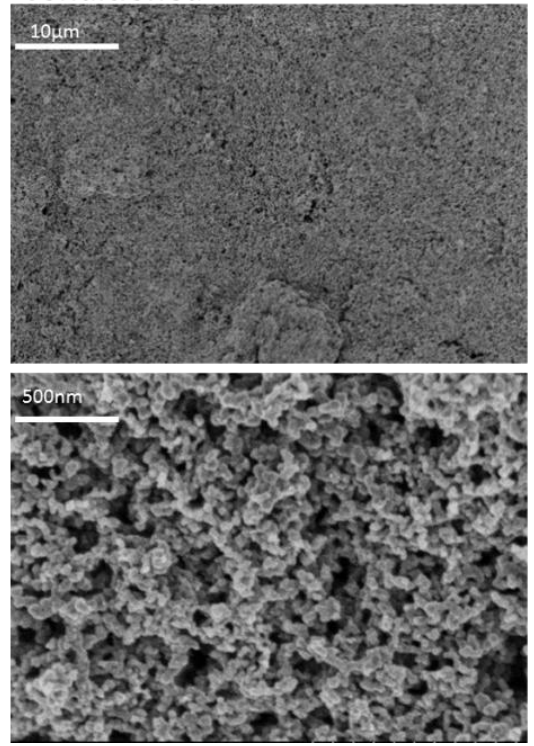

Aged after AST
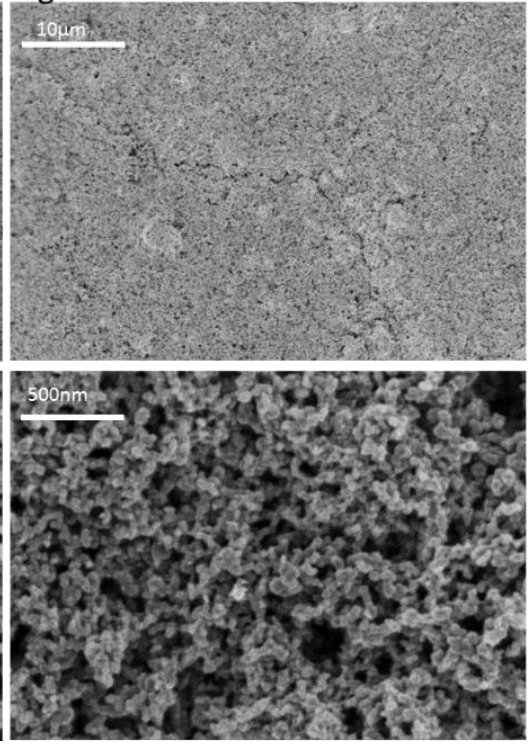

\section{Corroded after AST}
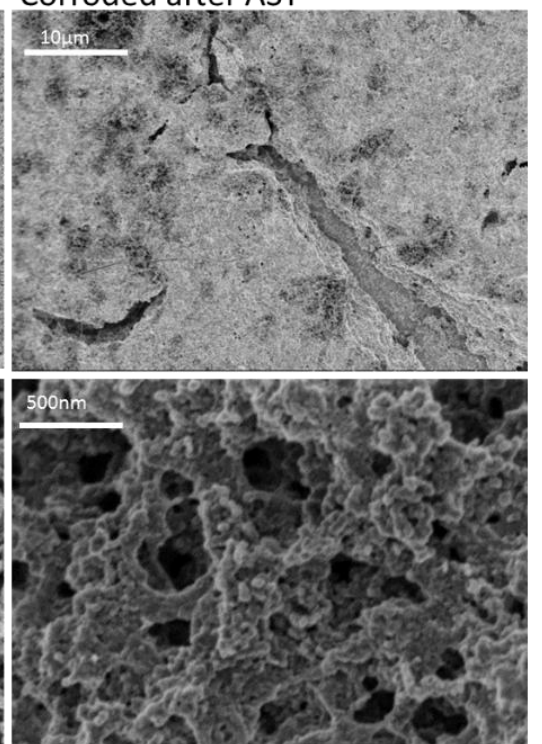

Figure 9. SEM images at two different magnifications for conditioned, aged after AST and corroded after AST HSAC CCM.
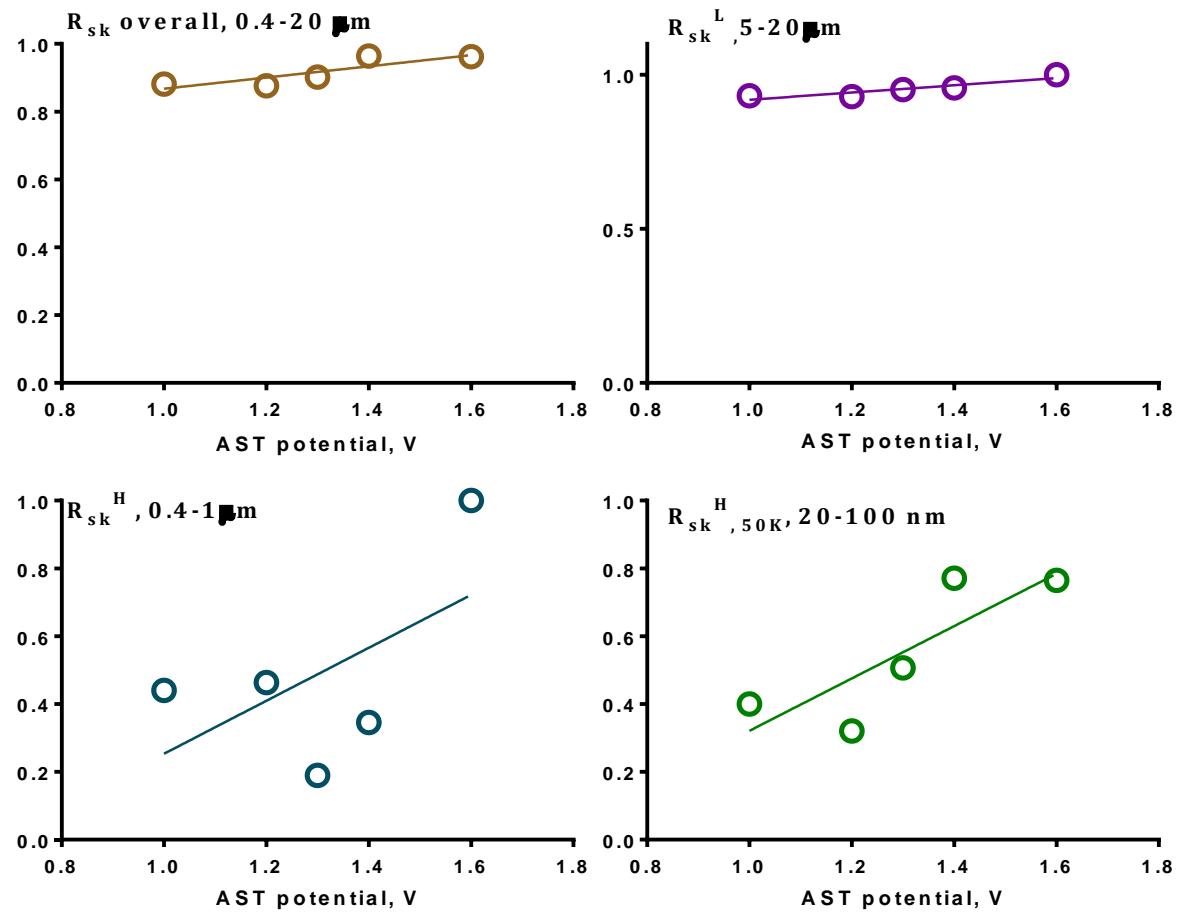

Figure 10. Skewness at four different scales as a function of AST UPL. 

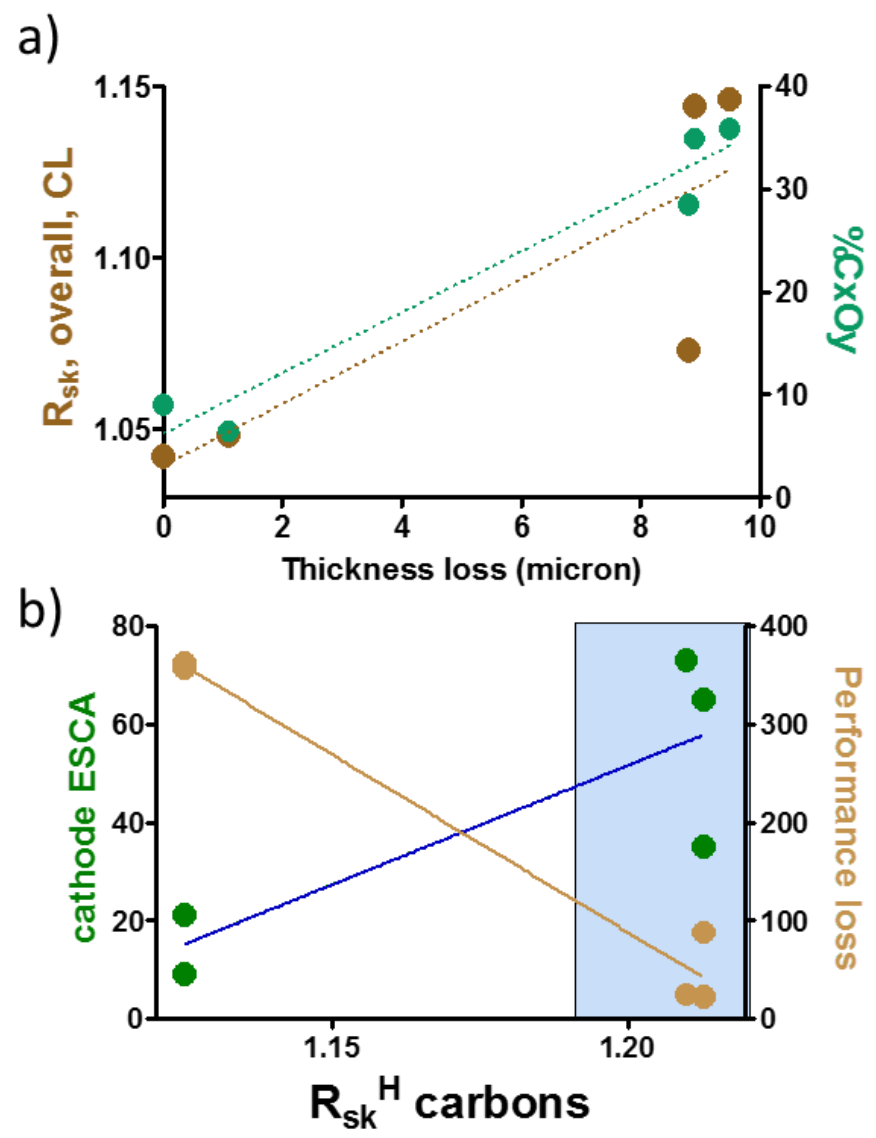

Figure 11. Morphology-to-performance correlations. Overall skewness of CCMs and amount of surface oxides as a function of thickness loss (a). Cathode ESCA and performance loss in V as a function of micro skewness(b). The durability window of $15 \%$ losses is highlighted. 


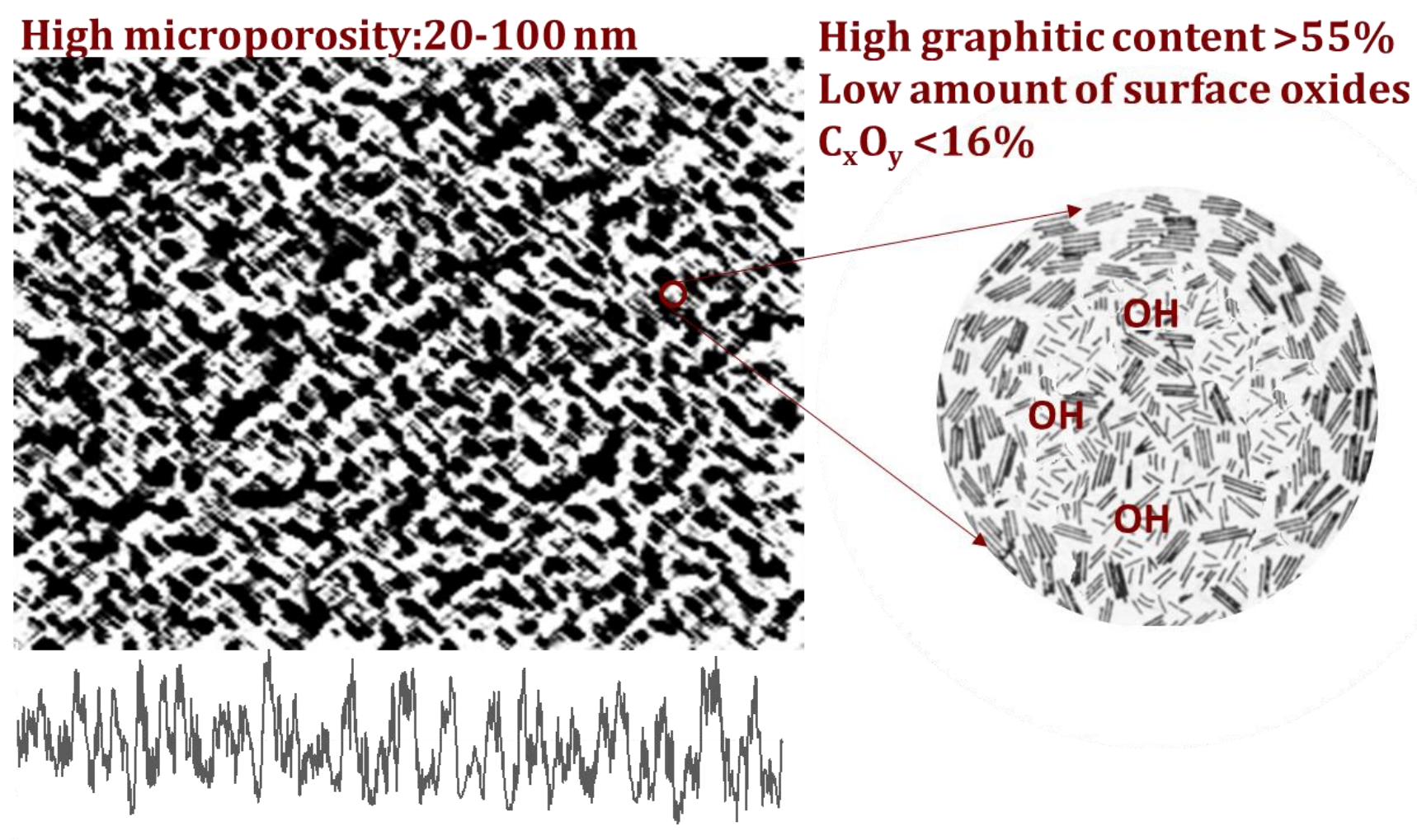

Figure 12. Structure of carbon support allowing catalyst layers that meet durability windows of 15\% loss: uniform high micro-porous morphologies with high graphitic content and low amount of surface oxides. 

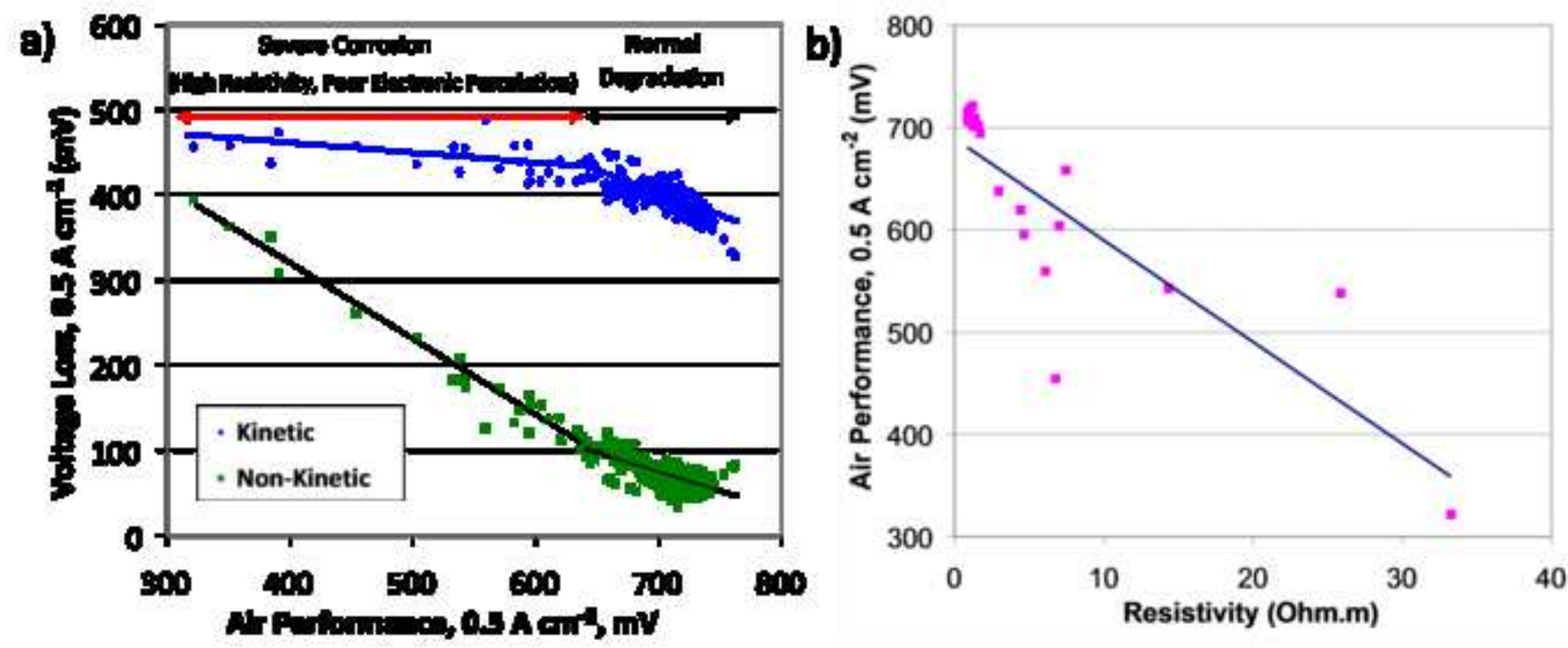


\section{Nafion ${ }^{\circledR} 1100 \mathrm{EW}$ $\mathrm{CF}_{2}: \mathrm{CF}=9$

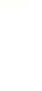

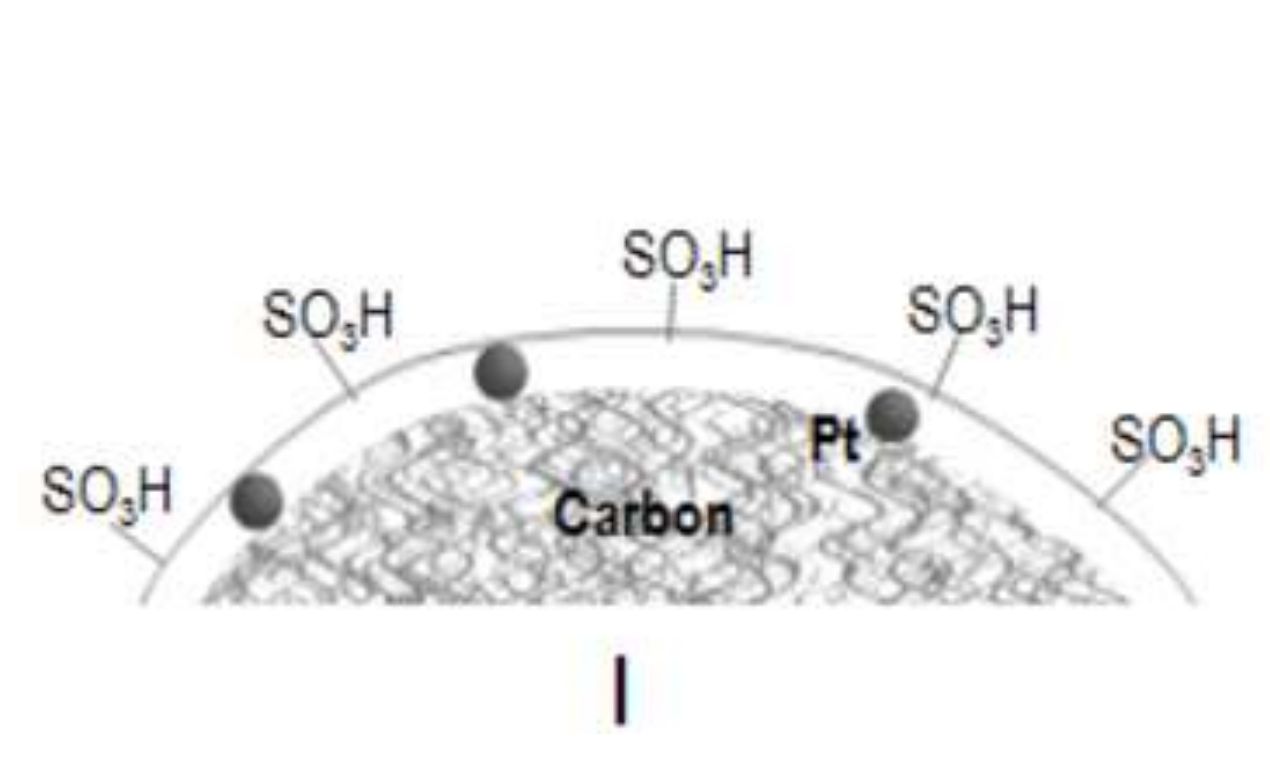

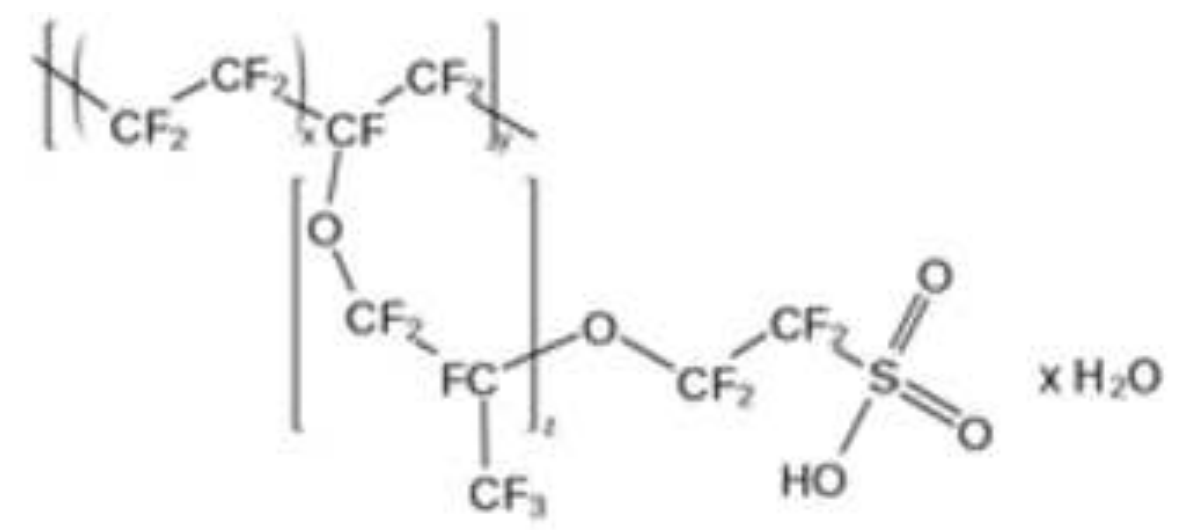

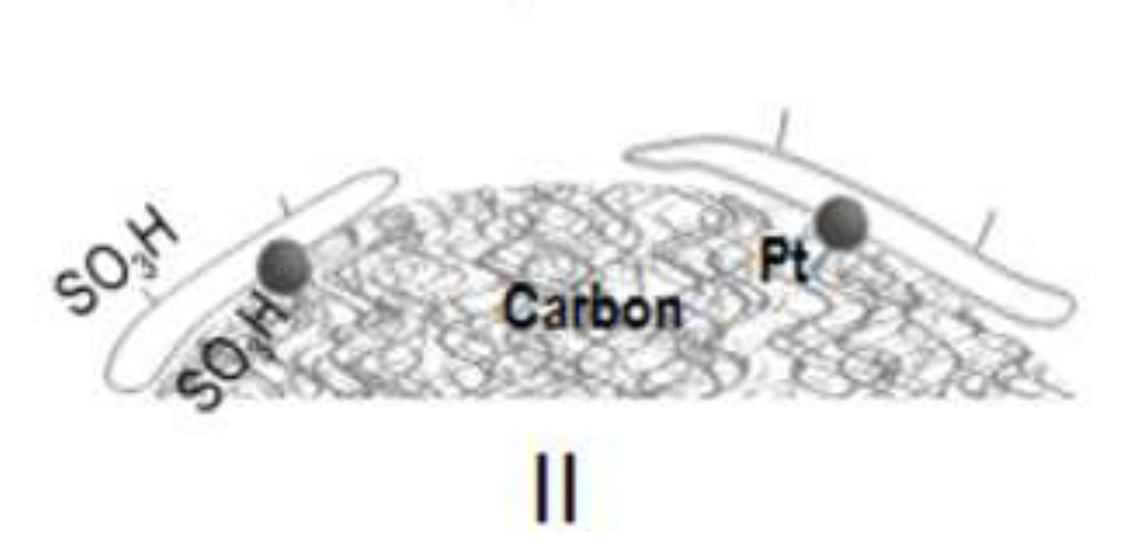



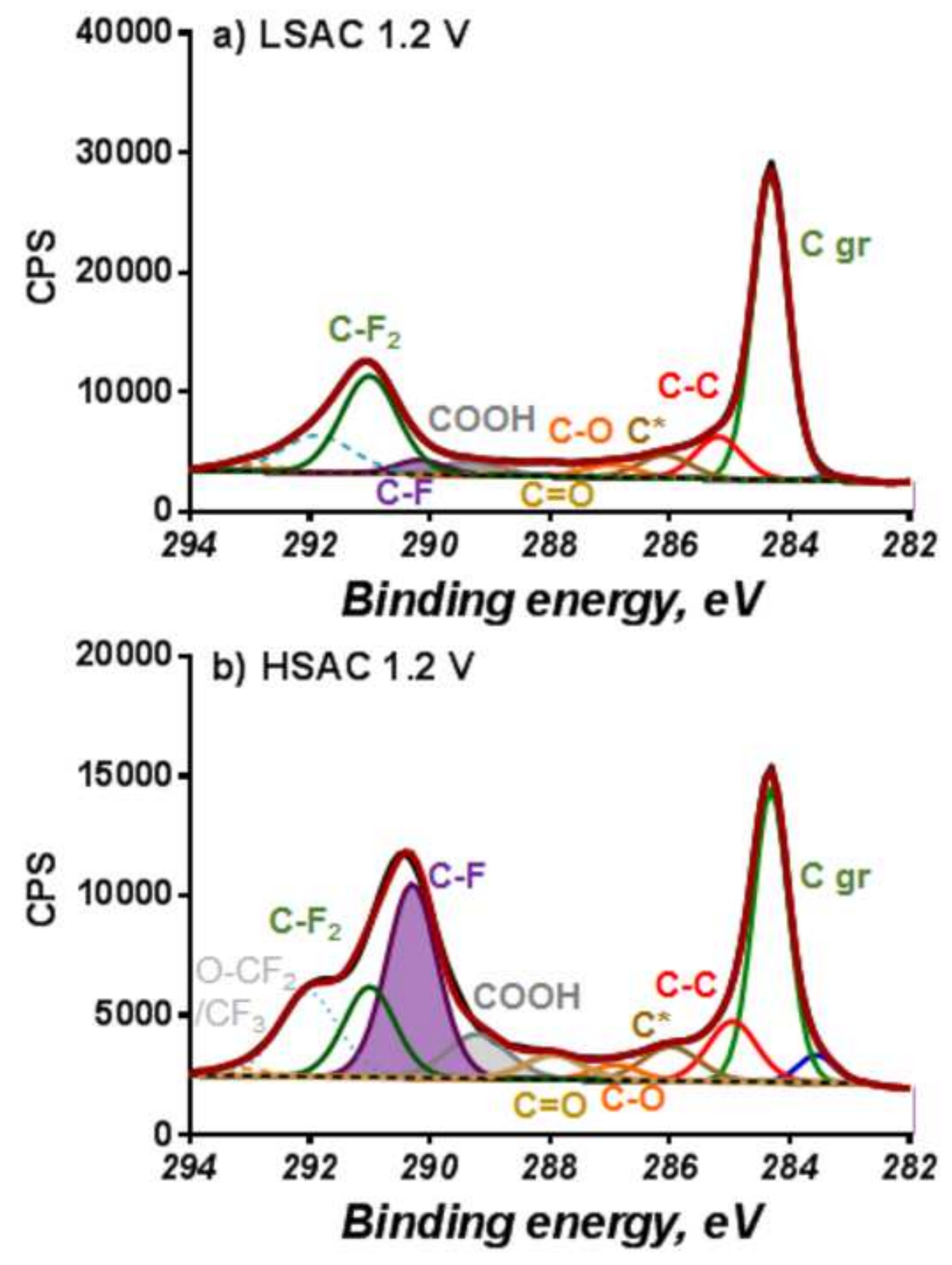

Figure(s) 


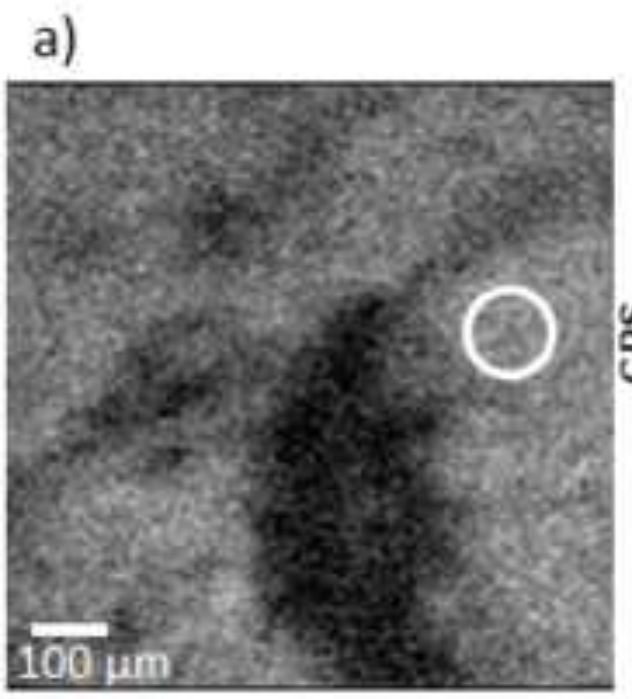

c)

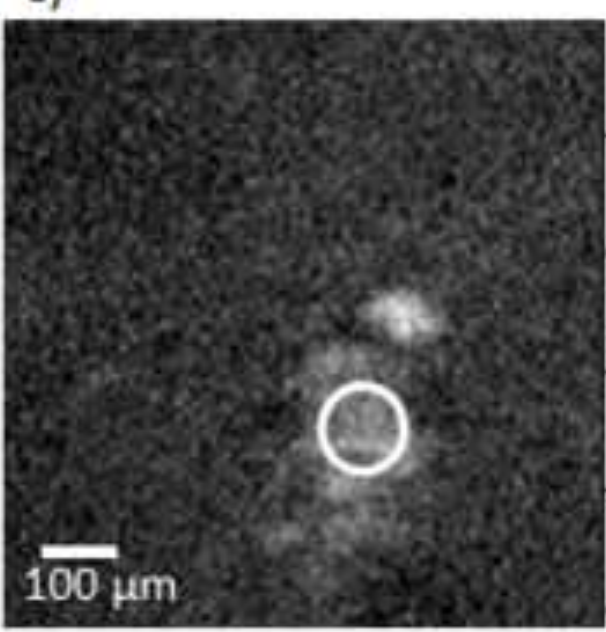

b)

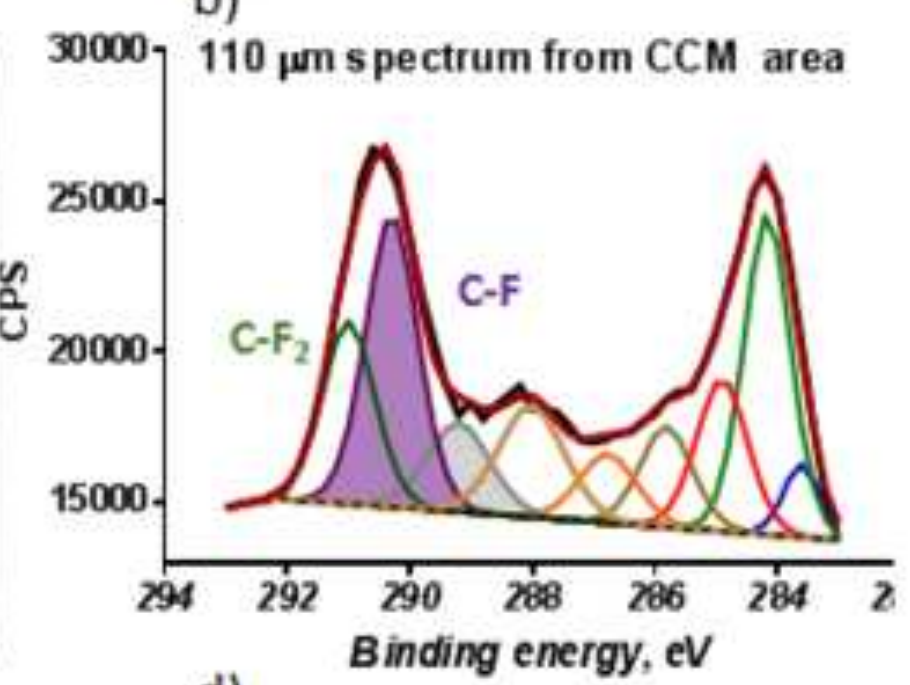

d)

$100000110 \mu \mathrm{m}$ spectrum from GDL area

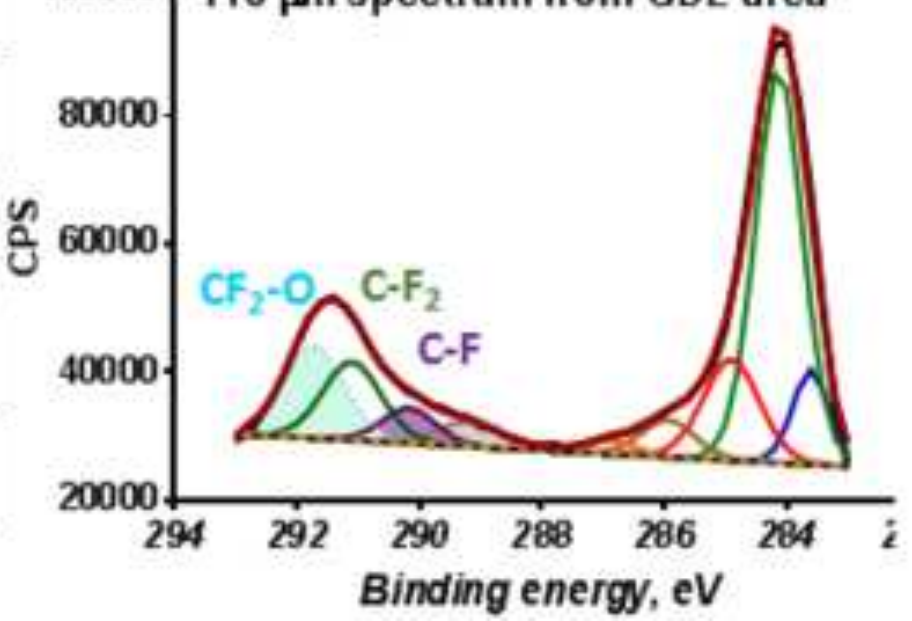

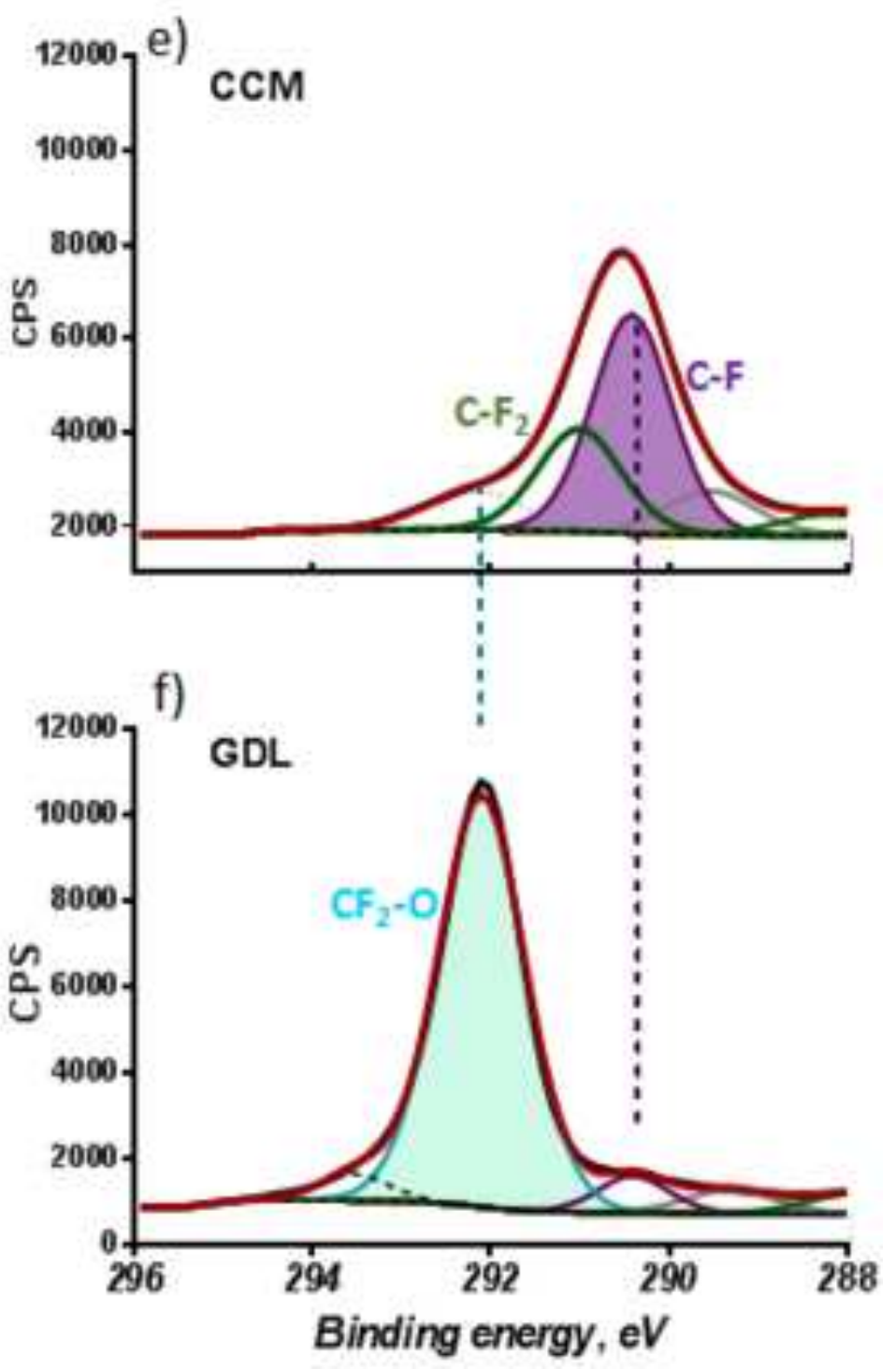



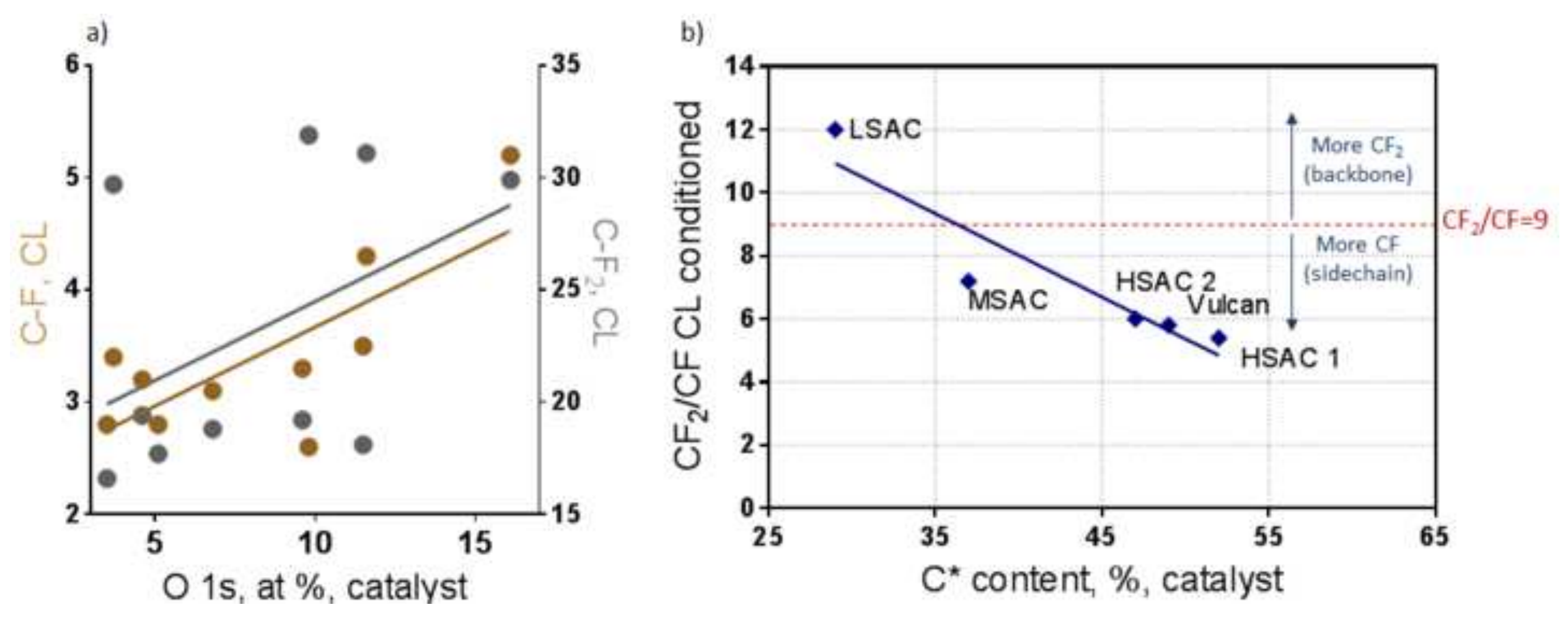

O $1 \mathrm{~s}$, at $\%$, catalyst

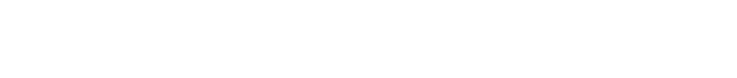



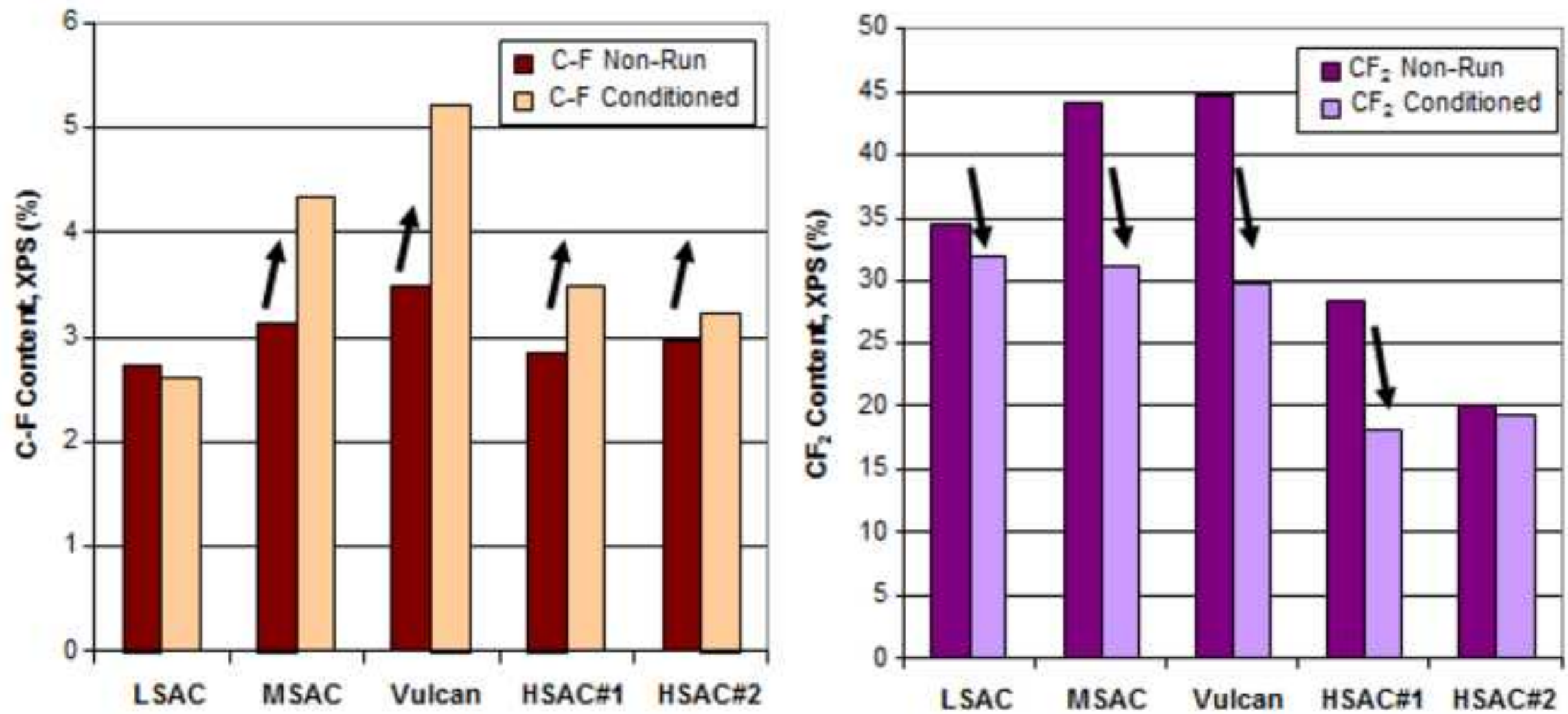
Conditioned
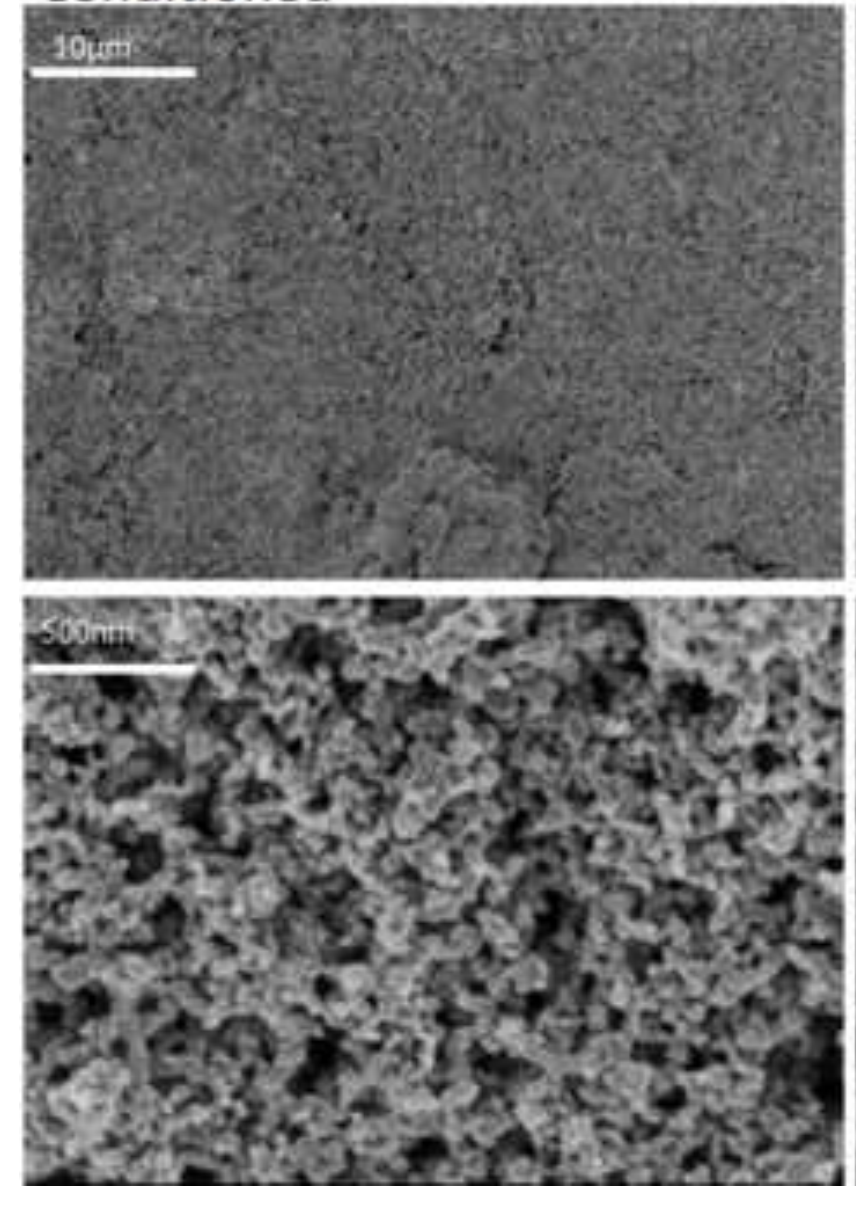

Aged after AST
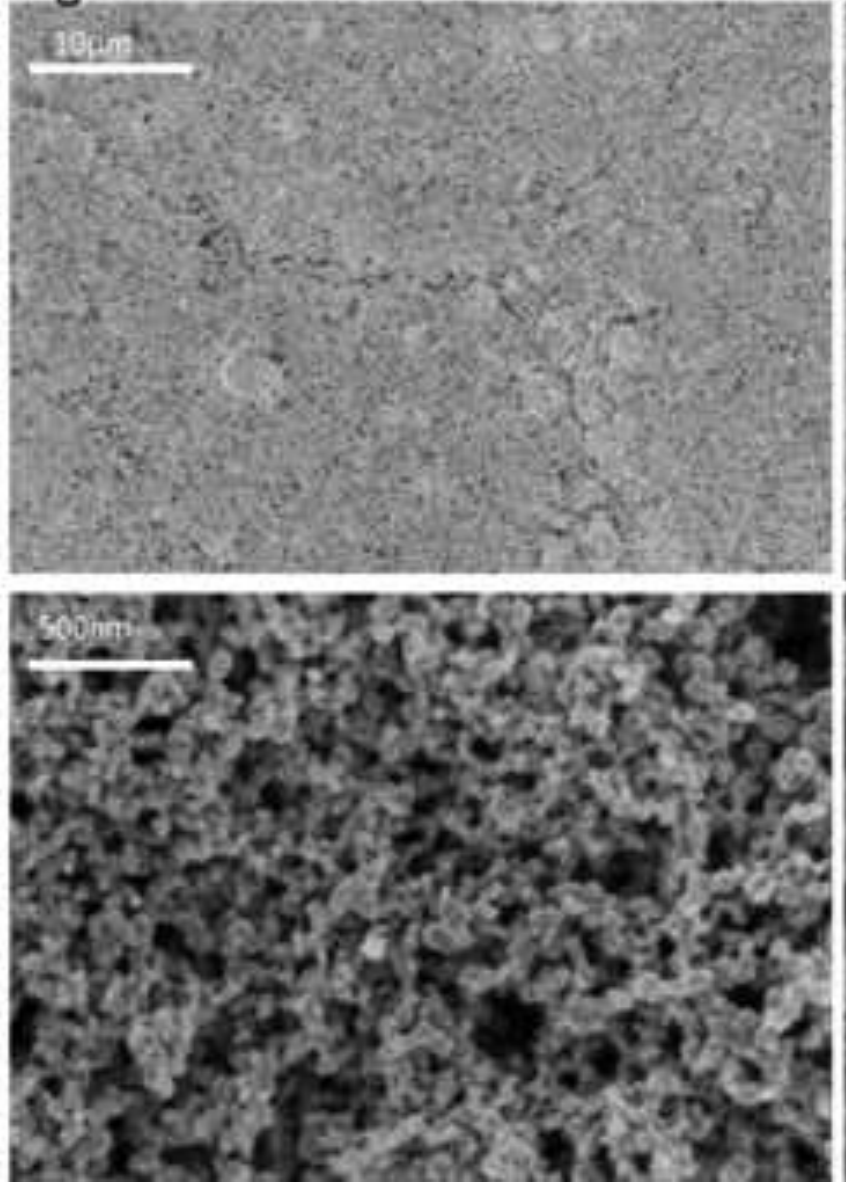

Corroded after AST

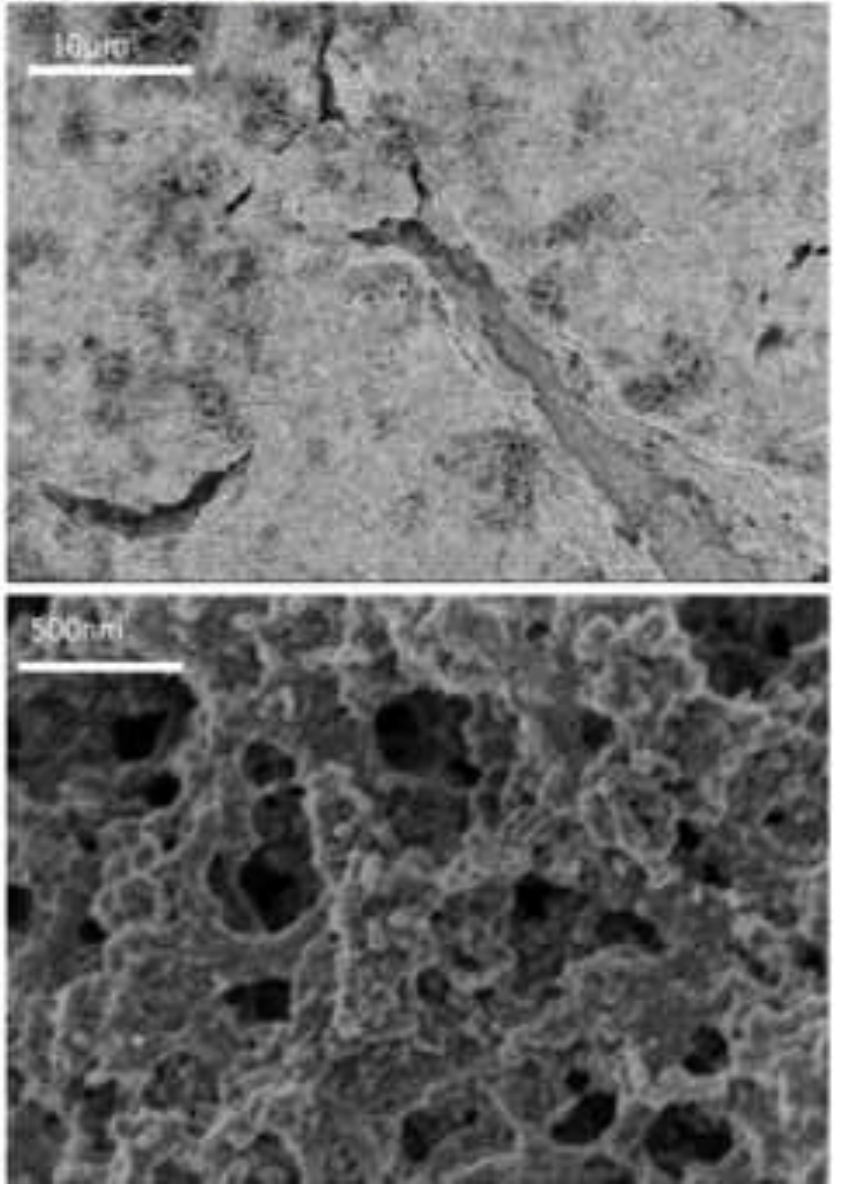



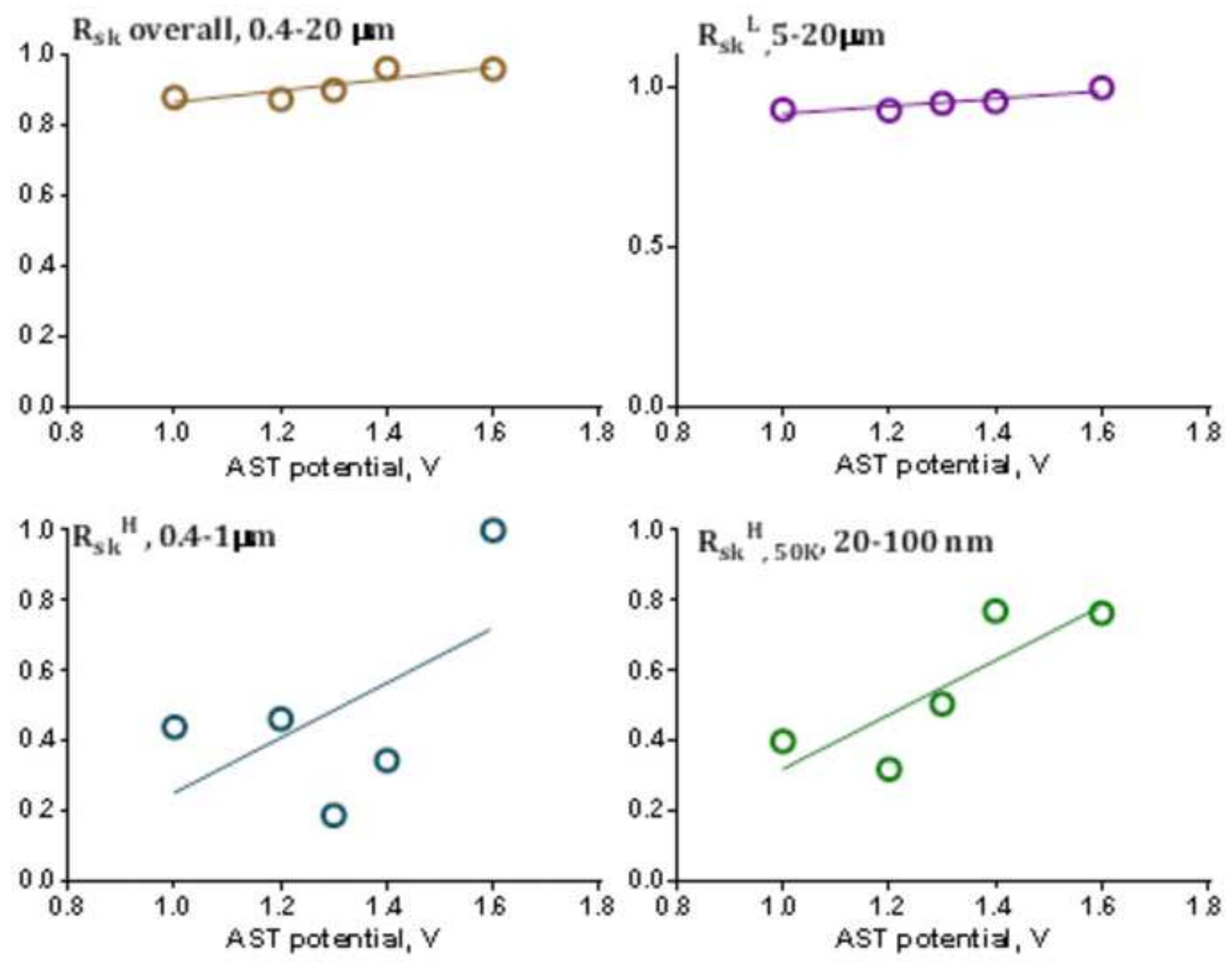
High microporosity:20-100 nm

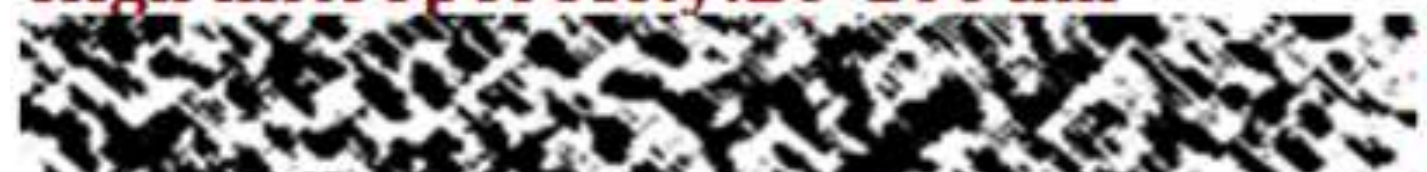

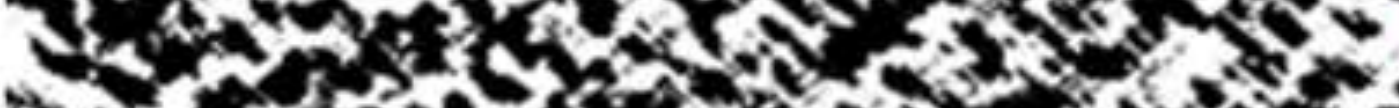

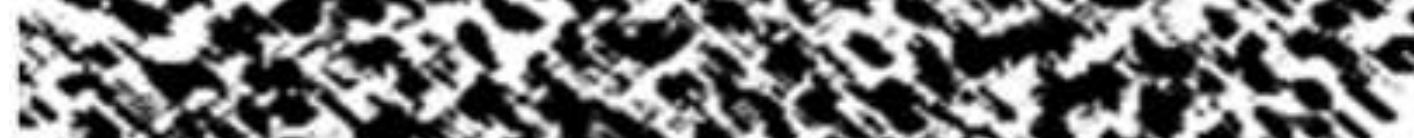
s.j.

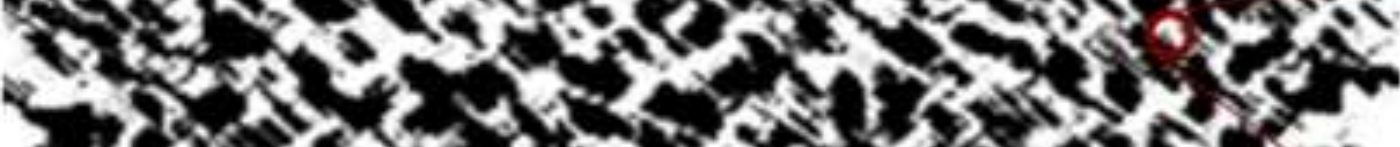

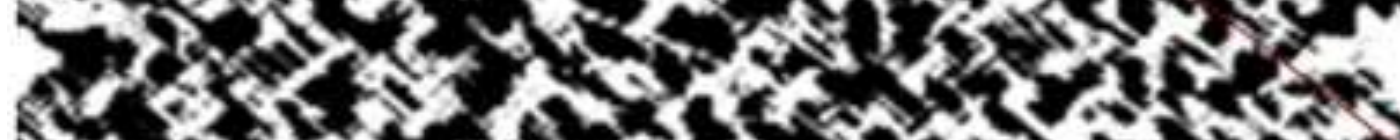

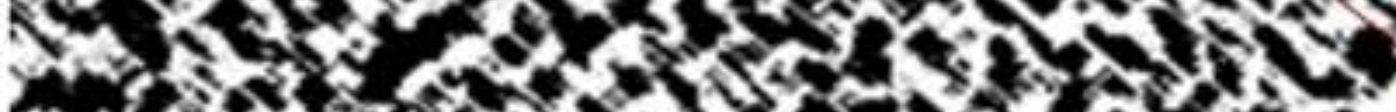

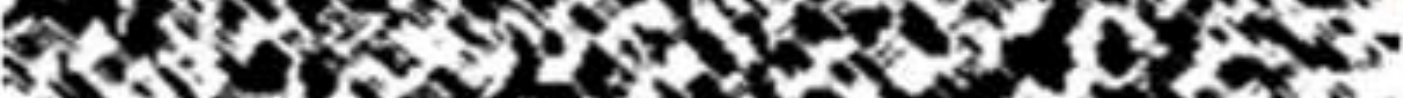

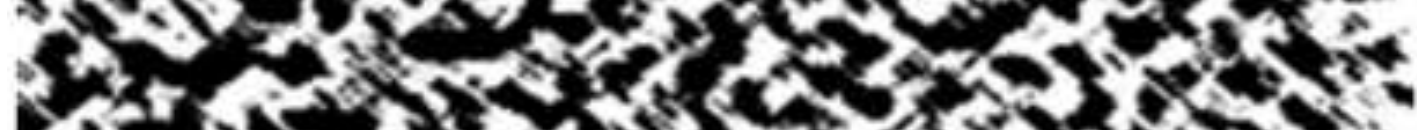

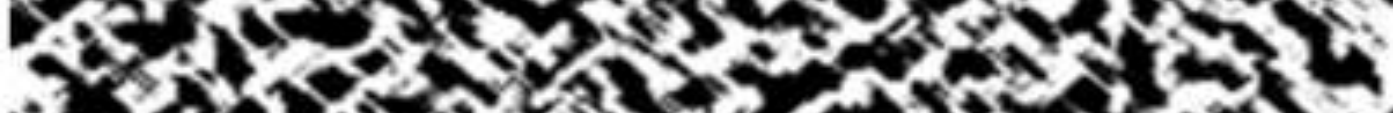

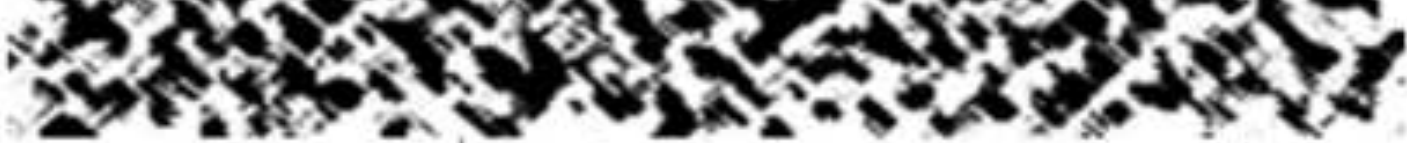

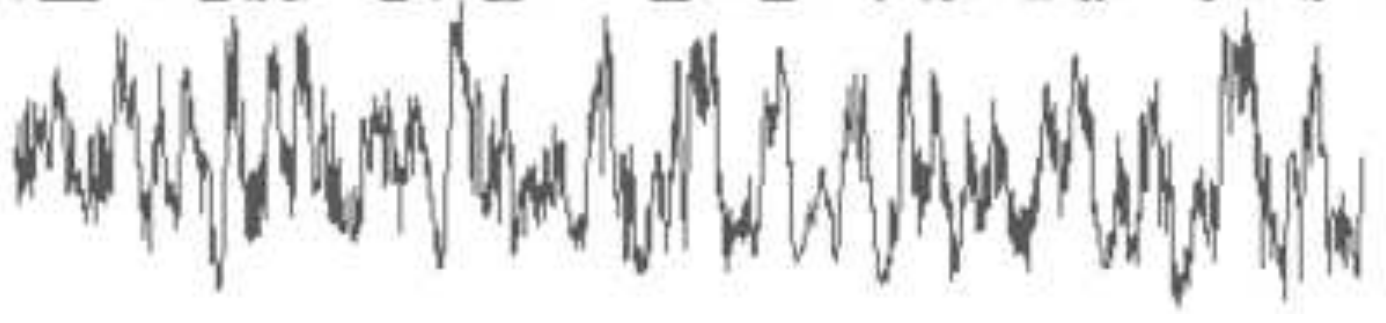

High graphitic content $>55 \%$ Low amount of surface oxides $\mathrm{C}_{\mathrm{x}} \mathrm{O}_{\mathrm{y}}<16 \%$

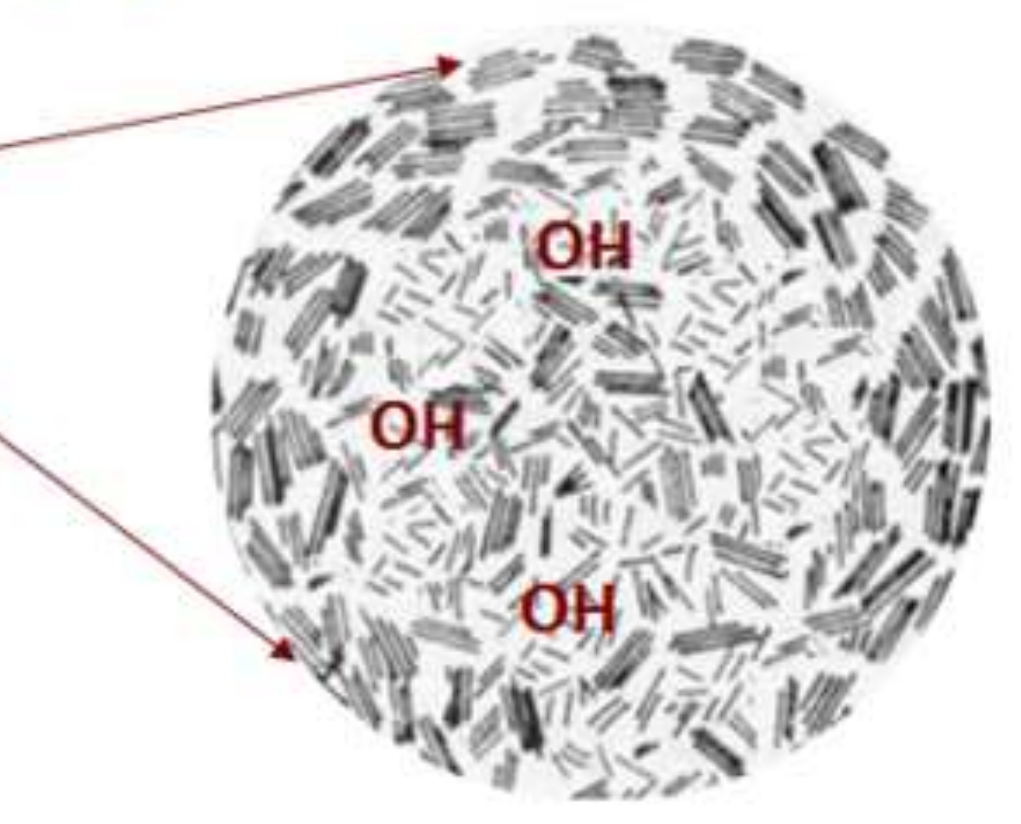

